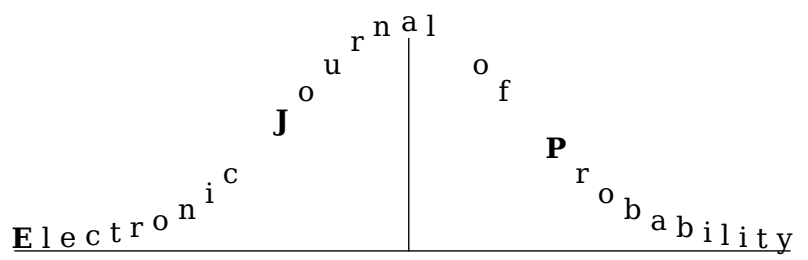

Electron. J. Probab. 26 (2021), article no. 87, 1-33.

ISSN: 1083-6489 https://doi.org/10.1214/21-EJP646

\title{
High-dimensional central limit theorems for a class of particle systems*
}

\author{
Jian Song $^{\dagger} \quad$ Jianfeng Yao $^{\ddagger} \quad$ Wangjun Yuan $^{\S}$
}

\begin{abstract}
We consider a class of particle systems that generalizes the eigenvalues of a class of matrix-valued processes, of which the empirical measures converge to deterministic measures as the dimension goes to infinity. In this paper, we obtain central limit theorems (CLTs) to characterize the fluctuations of the empirical measures around the limit measures by using stochastic calculus. As applications, CLTs for Dyson's Brownian motion and the eigenvalues of Wishart process are recovered under slightly more general initial conditions, and a CLT for the eigenvalues of a symmetric matrixvalued Ornstein-Uhlenbeck process is obtained.
\end{abstract}

Keywords: Dyson's Brownian motion; Wishart process; particle system; squared Bessel particle system; central limit theorem; matrix-valued Ornstein-Uhlenbeck process.

MSC2020 subject classifications: $60 \mathrm{H} 15 ; 60 \mathrm{~F} 05$.

Submitted to EJP on March 25, 2020, final version accepted on May 22, 2021.

\section{Introduction}

Recently general stochastic differential equations (SDEs) on the group of symmetric matrices have attracted much interest. A prominent example is the process introduced in Graczyk and Małecki (2013) as the solution of the following matrix-valued SDE,

$$
d X_{t}^{N}=g_{N}\left(X_{t}^{N}\right) d B_{t} h_{N}\left(X_{t}^{N}\right)+h_{N}\left(X_{t}^{N}\right) d B_{t}^{\top} g_{N}\left(X_{t}^{N}\right)+b_{N}\left(X_{t}^{N}\right) d t, \quad t \geq 0 .
$$

Here, $B_{t}$ is a matrix-valued Brownian motion of dimension $N \times N$, and the continuous functions $g_{N}, h_{N}, b_{N}: \mathbb{R} \rightarrow \mathbb{R}$ act on the spectrum of $X_{t}^{N}$ (a function $f$ acts on the

*J. Song is supported by Shandong University grant 11140089963041 and National Natural Science Foundation of China grant 12071256. J. Yao is supported by HKSAR-RGC-Grant GRF-17307319.

${ }^{\dagger}$ Research Center for Mathematics and Interdisciplinary Sciences, Shandong University, Qingdao, Shandong, 266237, China and School of Mathematics, Shandong University, Jinan, Shandong, 250100, China. E-mail: txj song@hotmail.com

${ }^{\ddagger}$ Department of Statistics and Actuarial Science, The University of Hong Kong.

E-mail: jeffyao@hku.hk

${ }^{\S}$ Department of Mathematics, The University of Hong Kong.

E-mail: ywangjun@connect.hku.hk 
spectrum of a symmetric matrix $X=\sum_{j=1}^{N} \alpha_{j} u_{j} u_{j}^{\top}$ with eigenvalues $\left(\alpha_{j}\right)$ and eigenvectors $\left(u_{j}\right)$ if $\left.f(X)=\sum_{j=1}^{N} f\left(\alpha_{j}\right) u_{j} u_{j}^{\top}\right)$. The matrix-valued process (1.1) extends several well-known processes such as the celebrated symmetric matrix-valued Brownian motion (Dyson, 1962), Wishart process (Bru, 1991), and the symmetric matrix-valued OrnsteinUhlenbeck processes (Chan, 1992).

Let $\left\{\lambda_{i}^{N}(t)\right\}_{1 \leq i \leq N}$ be the eigenvalues of $X_{t}^{N}$. According to Theorem 3 in Graczyk and Małecki (2013), if the initial eigenvalues $\left\{\lambda_{i}^{N}(0)\right\}_{1 \leq i \leq N}$ are all distinct, then before the first collision time $\tau_{N}=\inf \left\{t>0: \exists i \neq j, \lambda_{i}(t)=\lambda_{j}(t)\right\}$, the eigenvalue processes satisfy the following system of SDEs: for $1 \leq i \leq N$,

$$
d \lambda_{i}^{N}(t)=2 g_{N}\left(\lambda_{i}^{N}(t)\right) h_{N}\left(\lambda_{i}^{N}(t)\right) d W_{i}(t)+\left(b_{N}\left(\lambda_{i}^{N}(t)\right)+\sum_{j: j \neq i} \frac{G_{N}\left(\lambda_{i}^{N}(t), \lambda_{j}^{N}(t)\right)}{\lambda_{i}^{N}(t)-\lambda_{j}^{N}(t)}\right) d t,
$$

where $\left\{W_{i}, 1 \leq i \leq N\right\}$ are independent Brownian motions and

$$
G_{N}(x, y)=g_{N}^{2}(x) h_{N}^{2}(y)+g_{N}^{2}(y) h_{N}^{2}(x) .
$$

In Graczyk and Małecki $(2013,2014)$, some other conditions on the coefficient functions were imposed to ensure that (1.2) has a unique strong solution and the collision time $\tau_{N}$ is infinite almost surely.

Let $L_{N}(t)$ be the empirical measure of the eigenvalues $\left\{\lambda_{i}^{N}(t)\right\}_{1 \leq i \leq N}$, that is

$$
L_{N}(t)=\frac{1}{N} \sum_{i=1}^{N} \delta_{\lambda_{i}^{N}(t)}
$$

In connection with the theory of random matrices, it is of interest to investigate possible limits of these empirical measures $\left\{L_{N}(t), t \in[0, T]\right\}$ when $N$ grows to infinity (highdimensional limits). An early result is the derivation of the Wigner semi-circle law as the only equilibrium point (with finite moments of all orders) of the equation satisfied by the limit of eigenvalue empirical measure processes in Chan (1992), where the entries of the symmetric matrix-valued processes are independent Ornstein-Uhlenbeck processes. The results were later generalized in Rogers and Shi (1993) to the following interacting particle system

$$
d X_{i}=\sqrt{\frac{2 \alpha}{N}} d B_{i}+\left(-\theta X_{i}+\frac{\alpha}{N} \sum_{j: j \neq i} \frac{1}{X_{i}-X_{j}}\right) d t, \quad 1 \leq i \leq N, \quad t \geq 0 .
$$

Cépa and Lépingle (1997) further generalized these SDEs to

$$
d X_{i}=\sigma\left(X_{i}\right) d B_{i}+\left(b\left(X_{i}\right)+\sum_{j: j \neq i} \frac{\gamma}{X_{i}-X_{j}}\right) d t, \quad 1 \leq i \leq N, \quad t \geq 0,
$$

with some coefficient functions $b, \sigma$ and constant $\gamma$. Another important case is the Marčenko-Pastur law for the eigenvalue empirical measure processes derived in CabanalDuvillard and Guionnet (2001). In the framework of free probability theory, the semicircle law and Marčenko-Pastur law arise naturally as a consequence of the central limit theorem (CLT) for free random variables (Voiculescu (1991); Voiculescu et al. (1992)). We refer the interested reader to Hiai and Petz (2000); Mingo and Speicher (2017) and the references therein for this topic.

The eigenvalue SDEs (1.2) generalize the eigenvalue SDEs in Chan (1992) and Cabanal-Duvillard and Guionnet (2001), as well as the particle system in Rogers and 
Shi (1993). High-dimensional limits for these eigenvalue SDEs appeared very recently in Song et al. (2020) and Małecki and Pérez (2019). Particularly in the former article, it was proved that under proper conditions, $\left\{L_{N}(t), t \in[0, T]\right\}_{N \in \mathbb{N}}$ is relatively compact in $\left(C[0, T], M_{1}(\mathbb{R})\right)$ almost surely. Here $M_{1}(\mathbb{R})$ is the set of probability measures on $\mathbb{R}$ endowed with the topology induced by the weak convergence of measures.

Furthermore, any limit measure $\left\{\mu_{t}, t \in[0, T]\right\}$ from a converging subsequence satisfies

$$
\begin{aligned}
\int \frac{\mu_{t}(d x)}{z-x}= & \int \frac{\mu_{0}(d x)}{z-x}+\int_{0}^{t}\left[\int \frac{b(x)}{(z-x)^{2}} \mu_{s}(d x)\right] d s \\
& +\int_{0}^{t}\left[\iint \frac{G(x, y)}{(z-x)(z-y)^{2}} \mu_{s}(d x) \mu_{s}(d y)\right] d s, \quad \forall z \in \mathbb{C} \backslash \mathbb{R},
\end{aligned}
$$

with

$$
b(x)=\lim _{N \rightarrow \infty} b_{N}(x) \text { and } G(x, y)=\lim _{N \rightarrow \infty} N G_{N}(x, y),
$$

uniformly. To obtain the convergence of the relatively compact sequence of empirical measures $\left\{L_{N}(t), t \in[0, T]\right\}_{n \in \mathbb{N}}$, one would expect the solution of (1.4) to be unique. The uniqueness can be obtained under proper conditions for some symmetric matrix-valued processes, e.g., Dyson's Brownian motion (Anderson et al. (2010); Chan (1992); Rogers and Shi (1993)), matrix-valued Ornstein-Uhlenbeck process (Chan (1992); Rogers and Shi (1993)), Wishart process (Cabanal-Duvillard and Guionnet (2001)), self-similar processes (Song et al. (2020)), and Dyson's Brownian motion with a drift (Cépa and Lépingle (1997); Fontbona (2004); Li et al. (2020)). However, general conditions on the coefficient functions in (1.4) that guarantee the uniqueness of the solution are still unknown. In particular, Małecki and Pérez (2019) provided examples of (1.4) which have at least two solutions.

We aim to study the fluctuations of $\left\{L_{N}(t), t \in[0, T]\right\}$ of the eigenvalue SDE (1.2) around the limit $\left\{\mu_{t}, t \in[0, T]\right\}$ as $N \rightarrow \infty$, up to considering a subsequence. Indeed, we shall consider the following general particle system introduced in Graczyk and Małecki (2014) which includes (1.2) as a particular case: for $1 \leq i \leq N$,

$$
d x_{i}^{N}(t)=\sigma^{N}\left(x_{i}^{N}(t)\right) d W_{i}(t)+\left(b_{N}\left(x_{i}^{N}(t)\right)+\sum_{j: j \neq i} \frac{H_{N}\left(x_{i}^{N}(t), x_{j}^{N}(t)\right)}{x_{i}^{N}(t)-x_{j}^{N}(t)}\right) d t, t \geq 0,
$$

with $H_{N}(x, y)$ being a symmetric function. Under proper conditions, the existence and uniqueness of the non-colliding strong solution was obtained in Graczyk and Małecki (2014), and it was shown in Song et al. (2020) that the family of empirical measures $\left\{L_{N}(t), t \in[0, T]\right\}_{n \in \mathbb{N}}$ given by

$$
L_{N}(t)=\frac{1}{N} \sum_{i=1}^{N} \delta_{x_{i}^{N}(t)}
$$

is tight almost surely, and any limit $\left\{\mu_{t}, t \in[0, T]\right\}$ satisfies

$$
\begin{aligned}
\int \frac{\mu_{t}(d x)}{z-x}= & \int \frac{\mu_{0}(d x)}{z-x}+\int_{0}^{t}\left[\int \frac{b(x)}{(z-x)^{2}} \mu_{s}(d x)\right] d s+\int_{0}^{t}\left[\int \frac{\sigma(x)^{2}}{(z-x)^{3}} \mu_{s}(d x)\right] d s \\
& +\int_{0}^{t}\left[\iint \frac{H(x, y)}{(z-x)(z-y)^{2}} \mu_{s}(d x) \mu_{s}(d y)\right] d s, \quad \forall z \in \mathbb{C} \backslash \mathbb{R},
\end{aligned}
$$

where the continuous functions $b(x), \sigma(x)$ and $H(x, y)$ are the uniform limits of $b_{N}(x)$, $\sigma^{N}(x)$ and $N H_{N}(x, y)$, respectively. 
Up to considering a subsequence, we can assume, without loss of generality, the uniqueness of the limit process $\left\{\mu_{t}, t \in[0, T]\right\}$. Consider the random fluctuations,

$$
\mathcal{L}_{t}^{N}(f)=N\left\langle f, L_{N}(t)-\mu_{t}\right\rangle=\sum_{i=1}^{N} f\left(\lambda_{i}^{N}(t)\right)-N\left\langle f, \mu_{t}\right\rangle, \quad N \in \mathbb{N},
$$

where $f$ belongs to an appropriate space of test functions and

$$
\langle f, \mu\rangle=\int f(x) \mu(d x),
$$

for a measure $\mu$ on $\mathbb{R}$.

The main purpose of the paper is to find, as $N \rightarrow \infty$, a Gaussian limit for the centered process

$$
\begin{aligned}
Q_{t}^{N}(f)= & \mathcal{L}_{t}^{N}(f)-\mathcal{L}_{0}^{N}(f)-\int_{0}^{t} \mathcal{L}_{s}^{N}\left(f^{\prime} b\right) d s-\frac{1}{2} \int_{0}^{t} \int f^{\prime \prime}(x)\left(\tilde{\sigma}^{2}(x)-H(x, x)\right) \mu_{s}(d x) d s \\
& -\int_{0}^{t} \mathcal{L}_{s}^{N}\left(\int \frac{f^{\prime}(x)-f^{\prime}(y)}{x-y} H(x, y) \mu_{s}(d x)\right) d s \\
& -\frac{N}{2} \int_{0}^{t} \iint \frac{f^{\prime}(x)-f^{\prime}(y)}{x-y} H(x, y)\left[L_{N}(s)(d x)-\mu_{s}(d x)\right]\left[L_{N}(s)(d y)-\mu_{s}(d y)\right] d s,
\end{aligned}
$$

where $\tilde{\sigma}(x)$ is the uniform limit of $\sqrt{N} \sigma^{N}(x)$.

To our best knowledge, the problem of fluctuations for empirical measures of eigenvalue processes was first studied in Cabanal-Duvillard (2001) and later extended in Anderson et al. (2010). More precisely, Cabanal-Duvillard (2001) considered Dyson's Brownian motion and Wishart process with null initial condition, and established CLT for polynomial test functions; (Anderson et al., 2010, Theorem 4.3.20) extended the result for Dyson's Brownian motion by allowing bounded initial condition. Similar results were also obtained in Pérez-Abreu and Tudor (2007) and Perez-Abreu and Tudor (2009).

The rest of this paper is organized as follows.

In Section 2, we establish CLTs for the empirical measures of the general particle system (1.6). The space $\mathbb{F}$ of test functions is given by (2.1), which in general does not necessarily contain the set of all polynomials.

In Section 3, we apply the results in Section 2 to obtain CLTs for the eigenvalues of Wishart process in Section 3.2, for Dyson's Brownian motion in Section 3.3, and for the eigenvalues of symmetric matrix-valued Ornstein-Uhlenbeck process in Section 3.4, respectively. Note that in these three cases, one key ingredient is the following uniform bound for the moments of the eigenvalue processes

$$
\mathbb{E}\left[\sup _{t \in[0, T]}\left\langle|x|^{p}, L_{N}(t)\right\rangle\right] \leq C^{p},
$$

which enables us to obtain more precise CLTs for a larger class of test functions (in comparison with $\mathbb{F}$ given in (2.1)). For Dyson's Brownian motion, such bound was obtained in Anderson et al. (2010) by using the explicit joint density function of the eigenvalues (see Lemma 4.3.17 therein). This density approach is developed here in full detail for the Wishart case (Section 3.2). However, for more general particle systems that we consider in this paper, as the joint density functions are not available, some new tool is needed in order to derive the uniform moment bound (1.11). We thus establish in Section 3.1 a comparison principle for the particle system (1.6), which is also of interest 
in itself. This comparison principle allows to obtain the uniform bound (1.11) and then extend the CLTs to a larger class of particle systems (Corollaries 3.7, 3.10 and 3.12).

Furthermore, due to the special structures of Wishart process, Dyson's Brownian motion, and matrix-valued Ornstein-Uhlenbeck process, we are able to directly characterize the fluctuations $\left\{\mathcal{L}_{t}\left(x^{n}\right), t \in[0, T]\right\}_{n \in \mathbb{N}}$, where $\mathcal{L}_{t}\left(x^{n}\right)$ is the limit of $\mathcal{L}_{t}^{N}\left(x^{n}\right)$, by recursive formulas (see Theorems 3.5, 3.8, 3.11 and the remarks thereafter). For Dyson's Brownian motion and Wishart process, our CLTs provide extensions of the existing results in Cabanal-Duvillard (2001) and Anderson et al. (2010), while the CLT obtained in Section 3.4 for the eigenvalue processes of matrix-valued Ornstein-Uhlenbeck process seems to be new.

Finally, some useful lemmas are provided in Appendix A.

\section{Central limit theorems}

In this section, we derive a central limit theorem for the empirical measure (1.7) of the particle system (1.6).

Let $C_{b}^{k}(\mathbb{R})$ be the space of bounded continuous real-valued functions with bounded continuous derivatives (up to the order $k$ ). Recall that the continuous functions $b(x)$, $\sigma(x)$ and $H(x, y)$ are the uniform limits of $b_{N}(x), \sigma^{N}(x)$ and $N H_{N}(x, y)$, respectively, and $Q_{t}^{N}(f)$ is defined in (1.10). We use the following space of test functions

$$
\begin{gathered}
\mathbb{F}=\left\{f \in C_{b}^{2}(\mathbb{R}):\left\|f^{\prime}(x) b(x)\right\|_{L^{\infty}(\mathbb{R})}<\infty, \quad\left\|\frac{f^{\prime}(x)-f^{\prime}(y)}{x-y} H(x, y)\right\|_{L^{\infty}\left(\mathbb{R}^{2}\right)}<\infty,\right. \\
\left.\left\|f^{\prime}(x) \tilde{\sigma}(x)\right\|_{L^{\infty}(\mathbb{R})}<\infty, \quad\left\|f^{\prime \prime}(x) \tilde{\sigma}^{2}(x)\right\|_{L^{\infty}(\mathbb{R})}<\infty\right\},
\end{gathered}
$$

where the continuous function $\tilde{\sigma}(x)$ is the uniform limit of $\sqrt{N} \sigma^{N}(x)$.

Remark 2.1. For Dyson's Brownian motion, $\sigma^{N}(x)=\frac{\sqrt{2}}{\sqrt{N}}, b_{N}(x)=0, H_{N}(x, y)=\frac{1}{N}$ (see also Section 3.3). Thus, the space $\mathbb{F}$ of test functions is $C_{b}^{2}(\mathbb{R})$. For Wishart process, $\sigma^{N}(x)=2 \frac{\sqrt{x}}{\sqrt{N}}, b_{N}(x)=\frac{P}{N}, H_{N}(x, y)=\frac{x+y}{N}$ (see also Section 3.2), the limits of the coefficient functions are $\tilde{\sigma}(x)=2 \sqrt{x}, b(x)=c$ and $H(x, y)=x+y$. In this case, $\mathbb{F}$ is the set of functions $f(x)$ in $C_{b}^{2}(\mathbb{R})$ satisfying $\left\|\sqrt{x} f^{\prime}(x)\right\|_{L^{\infty}(\mathbb{R})}<\infty$ and $\left\|x f^{\prime \prime}(x)\right\|_{L^{\infty}(\mathbb{R})}<\infty$. For Ornstein-Uhlenbeck process, $\sigma^{N}(x)=\frac{1}{\sqrt{N}}, b_{N}(x)=-\frac{1}{2} x, H_{N}(x, y)=\frac{1}{2 N}$ (see also Section 3.4), and correspondingly $\tilde{\sigma}(x)=1, b(x)=-\frac{1}{2} x$ and $H(x, y)=\frac{1}{2}$. In this case, $\mathbb{F}$ is the set of functions $f(x)$ in $C_{b}^{2}(\mathbb{R})$ satisfying $\left\|x f^{\prime}(x)\right\|_{L^{\infty}(\mathbb{R})}<\infty$. In Sections 3.2, 3.3 and 3.4, we will develop CLTs for a larger class of test functions which includes all polynomials for these three cases, respectively.

Theorem 2.2. Suppose that

$$
\begin{array}{r}
\lim _{N \rightarrow \infty} N\left\|b_{N}(x)-b(x)\right\|_{L^{\infty}(\mathbb{R})}=0 \\
\lim _{N \rightarrow \infty} N\left\|N H_{N}(x, y)-H(x, y)\right\|_{L^{\infty}\left(\mathbb{R}^{2}\right)}=0 .
\end{array}
$$

Also assume that (1.6) has a non-exploding and non-colliding strong solution, such that the sequence of the empirical measures $\left\{L_{N}(t), t \in[0, T]\right\}_{N \in \mathbb{N}}$ converges weakly to $\left\{\mu_{t}, t \in[0, T]\right\}$.

Then, for any $k \in \mathbb{N}$ and any $f_{1}, \ldots, f_{k} \in \mathbb{F},\left(Q_{t}^{N}\left(f_{1}\right), \ldots, Q_{t}^{N}\left(f_{k}\right)\right)_{t \in[0, T]}$ converges in distribution to a centered Gaussian process $\left(G_{t}\left(f_{1}\right), \ldots, G_{t}\left(f_{k}\right)\right)_{t \in[0, T]}$ with covariance

$$
\mathbb{E}\left[G_{t}\left(f_{i}\right) G_{s}\left(f_{j}\right)\right]=\int_{0}^{t \wedge s}\left\langle f_{i}^{\prime}(x) f_{j}^{\prime}(x) \tilde{\sigma}^{2}(x), \mu_{u}\right\rangle d u, \quad 1 \leq i, j \leq k .
$$


Proof. By Itô's formula and (1.6), for $f \in C^{2}(\mathbb{R})$,

$$
\begin{aligned}
d f\left(x_{i}^{N}(t)\right)= & f^{\prime}\left(x_{i}^{N}(t)\right) \sigma^{N}\left(x_{i}^{N}(t)\right) d W_{i}(t)+f^{\prime}\left(x_{i}^{N}(t)\right) b_{N}\left(x_{i}^{N}(t)\right) d t \\
& +\sum_{j: j \neq i} \frac{f^{\prime}\left(x_{i}^{N}(t)\right) H_{N}\left(x_{i}^{N}(t), x_{j}^{N}(t)\right)}{x_{i}^{N}(t)-x_{j}^{N}(t)} d t+\frac{1}{2} f^{\prime \prime}\left(x_{i}^{N}(t)\right)\left(\sigma^{N}\left(x_{i}^{N}(t)\right)\right)^{2} d t .
\end{aligned}
$$

Thus, we have, noting that $H_{N}(x, y)$ is symmetric,

$$
\begin{aligned}
& \left\langle f, L_{N}(t)\right\rangle=\frac{1}{N} \sum_{i=1}^{N} f\left(x_{i}^{N}(t)\right) \\
& =\frac{1}{N} \sum_{i=1}^{N} f\left(x_{i}^{N}(0)\right)+\frac{1}{N} \sum_{i=1}^{N} \int_{0}^{t} f^{\prime}\left(x_{i}^{N}(s)\right) \sigma^{N}\left(x_{i}^{N}(s)\right) d W_{i}(s) \\
& +\frac{1}{N} \sum_{i=1}^{N} \int_{0}^{t} f^{\prime}\left(x_{i}^{N}(s)\right) b_{N}\left(x_{i}^{N}(s)\right) d s+\frac{1}{N} \int_{0}^{t} \sum_{i \neq j}^{N} \frac{f^{\prime}\left(x_{i}^{N}(s)\right) H_{N}\left(x_{i}^{N}(s), x_{j}^{N}(s)\right)}{x_{i}^{N}(s)-x_{j}^{N}(s)} d s \\
& +\frac{1}{2 N} \sum_{i=1}^{N} \int_{0}^{t} f^{\prime \prime}\left(x_{i}^{N}(s)\right)\left(\sigma^{N}\left(x_{i}^{N}(s)\right)\right)^{2} d s \\
& =\frac{1}{N} \sum_{i=1}^{N} f\left(x_{i}^{N}(0)\right)+\frac{1}{N} \sum_{i=1}^{N} \int_{0}^{t} f^{\prime}\left(x_{i}^{N}(s)\right) \sigma^{N}\left(x_{i}^{N}(s)\right) d W_{i}(s) \\
& +\frac{1}{N} \sum_{i=1}^{N} \int_{0}^{t} f^{\prime}\left(x_{i}^{N}(s)\right) b_{N}\left(x_{i}^{N}(s)\right) d s+\frac{1}{2 N} \sum_{i=1}^{N} \int_{0}^{t} f^{\prime \prime}\left(x_{i}^{N}(s)\right)\left(\sigma^{N}\left(x_{i}^{N}(s)\right)\right)^{2} d s \\
& +\frac{1}{2 N} \int_{0}^{t} \sum_{i \neq j}^{N} \frac{f^{\prime}\left(x_{i}^{N}(s)\right)-f^{\prime}\left(x_{j}^{N}(s)\right)}{x_{i}^{N}(s)-x_{j}^{N}(s)} H_{N}\left(x_{i}^{N}(s), x_{j}^{N}(s)\right) d s \\
& =\left\langle f, L_{N}(0)\right\rangle+M_{f}^{N}(t)+\int_{0}^{t}\left\langle f^{\prime} b_{N}, L_{N}(s)\right\rangle d s+\frac{1}{2} \int_{0}^{t}\left\langle f^{\prime \prime}\left(\sigma^{N}\right)^{2}, L_{N}(s)\right\rangle d s \\
& +\frac{1}{2 N} \int_{0}^{t} \sum_{i, j=1}^{N} \frac{f^{\prime}\left(x_{i}^{N}(s)\right)-f^{\prime}\left(x_{j}^{N}(s)\right)}{x_{i}^{N}(s)-x_{j}^{N}(s)} H_{N}\left(x_{i}^{N}(s), x_{j}^{N}(s)\right) d s \\
& -\frac{1}{2 N} \int_{0}^{t} \sum_{i=1}^{N} f^{\prime \prime}\left(x_{i}^{N}(s)\right) H_{N}\left(x_{i}^{N}(s), x_{i}^{N}(s)\right) d s \\
& =\left\langle f, L_{N}(0)\right\rangle+M_{f}^{N}(t)+\int_{0}^{t}\left\langle f^{\prime} b_{N}, L_{N}(s)\right\rangle d s \\
& +\frac{1}{2} \int_{0}^{t} \int f^{\prime \prime}(x)\left(\left(\sigma^{N}(x)\right)^{2}-H_{N}(x, x)\right) L_{N}(s)(d x) d s \\
& +\frac{N}{2} \int_{0}^{t} \iint \frac{f^{\prime}(x)-f^{\prime}(y)}{x-y} H_{N}(x, y) L_{N}(s)(d x) L_{N}(s)(d y) d s,
\end{aligned}
$$

where we use the convention $\frac{f^{\prime}(x)-f^{\prime}(y)}{x-y}=f^{\prime \prime}(x)$ if $x=y$, and

$$
M_{f}^{N}(t)=\frac{1}{N} \sum_{i=1}^{N} \int_{0}^{t} f^{\prime}\left(x_{i}^{N}(s)\right) \sigma^{N}\left(x_{i}^{N}(s)\right) d W_{i}(s)
$$

is a local martingale with quadratic variation

$$
\left\langle M_{f}^{N}\right\rangle_{t}=\frac{1}{N^{2}} \sum_{i=1}^{N} \int_{0}^{t}\left(f^{\prime}\left(x_{i}^{N}(s)\right) \sigma^{N}\left(x_{i}^{N}(s)\right)\right)^{2} d s=\frac{1}{N} \int_{0}^{t}\left\langle\left|f^{\prime} \sigma^{N}\right|^{2}, L_{N}(s)\right\rangle d s .
$$


Recall that the limit measure $\mu_{t}$ satisfies (1.8). For $f \in \mathbb{F}$, under condition (2.2), one may apply the approach used in the proof of Theorem 1 in Małecki and Pérez (2019) (see also Theorem 2.2 in Song et al. (2020)) to get, noting that $\sigma(x) \equiv 0$,

$$
\left\langle f, \mu_{t}\right\rangle=\left\langle f, \mu_{0}\right\rangle+\int_{0}^{t}\left\langle f^{\prime} b, \mu_{s}\right\rangle d s+\frac{1}{2} \int_{0}^{t} \iint \frac{f^{\prime}(x)-f^{\prime}(y)}{x-y} H(x, y) \mu_{s}(d x) \mu_{s}(d y) d s
$$

Thus, (2.4) and (2.7) yield

$$
\begin{aligned}
& \mathcal{L}_{t}^{N}(f)=N\left\langle f, L_{N}(t)-\mu_{t}\right\rangle \\
& =N\left\langle f, L_{N}(0)-\mu_{0}\right\rangle+N M_{f}^{N}(t) \\
& +N \int_{0}^{t}\left(\left\langle f^{\prime} b_{N}, L_{N}(s)\right\rangle-\left\langle f^{\prime} b, \mu_{s}\right\rangle\right) d s \\
& +\frac{N}{2} \int_{0}^{t} \int f^{\prime \prime}(x)\left(\left(\sigma^{N}(x)\right)^{2}-H_{N}(x, x)\right) L_{N}(s)(d x) d s \\
& +\frac{N}{2} \int_{0}^{t} \iint \frac{f^{\prime}(x)-f^{\prime}(y)}{x-y}\left[N H_{N}(x, y) L_{N}(s)(d x) L_{N}(s)(d y)-H(x, y) \mu_{s}(d x) \mu_{s}(d y)\right] d s .
\end{aligned}
$$

The third term on the right-hand side of (2.8) can be written as

$$
\begin{aligned}
& N \int_{0}^{t}\left(\left\langle f^{\prime} b_{N}, L_{N}(s)\right\rangle-\left\langle f^{\prime} b, \mu_{s}\right\rangle\right) d s \\
= & N \int_{0}^{t}\left\langle f^{\prime} b_{N}-f^{\prime} b, L_{N}(s)\right\rangle d s+N \int_{0}^{t}\left\langle f^{\prime} b, L_{N}(s)-\mu_{s}\right\rangle d s \\
= & N \int_{0}^{t}\left\langle f^{\prime} b_{N}-f^{\prime} b, L_{N}(s)\right\rangle d s+\int_{0}^{t} \mathcal{L}_{s}^{N}\left(f^{\prime} b\right) d s
\end{aligned}
$$

Thus, by (2.2), we have

$$
\begin{aligned}
& \lim _{N \rightarrow \infty}\left|N \int_{0}^{t}\left(\left\langle f^{\prime} b_{N}, L_{N}(s)\right\rangle-\left\langle f^{\prime} b, \mu_{s}\right\rangle\right) d s-\int_{0}^{t} \mathcal{L}_{s}^{N}\left(f^{\prime} b\right) d s\right| \\
\leq & \lim _{N \rightarrow \infty} N \int_{0}^{t}\left|\left\langle f^{\prime} b_{N}-f^{\prime} b, L_{N}(s)\right\rangle\right| d s \\
\leq & \lim _{N \rightarrow \infty} N T\left\|f^{\prime}\right\|_{L^{\infty}(\mathbb{R})}\left\|b_{N}-b\right\|_{L^{\infty}(\mathbb{R})}=0 .
\end{aligned}
$$

For the fourth term on the right-hand side of (2.8),

$$
\begin{aligned}
& \frac{N}{2} \int_{0}^{t} \int f^{\prime \prime}(x)\left(\left(\sigma^{N}(x)\right)^{2}-H_{N}(x, x)\right) L_{N}(s)(d x) d s \\
= & \frac{1}{2} \int_{0}^{t} \int f^{\prime \prime}(x)\left(N\left(\sigma^{N}(x)\right)^{2}-\tilde{\sigma}^{2}(x)+H(x, x)-N H_{N}(x, x)\right) L_{N}(s)(d x) d s \\
& +\frac{1}{2} \int_{0}^{t} \int f^{\prime \prime}(x)\left(\tilde{\sigma}^{2}(x)-H(x, x)\right) L_{N}(s)(d x) d s .
\end{aligned}
$$

Noting that both convergences $\sqrt{N} \sigma^{N} \rightarrow \tilde{\sigma}$ and $N H_{N}(x, x) \rightarrow H(x, x)$ are uniform as 
$N \rightarrow \infty$, we have

$$
\begin{aligned}
& \mid \frac{N}{2} \int_{0}^{t} f^{\prime \prime}(x)\left(\left(\sigma^{N}(x)\right)^{2}-H_{N}(x, x)\right) L_{N}(s)(d x) d s \\
& \quad-\frac{1}{2} \int_{0}^{t} \int f^{\prime \prime}(x)\left(\tilde{\sigma}^{2}(x)-H(x, x)\right) \mu_{s}(d x) d s \mid \\
& \leq\left|\frac{1}{2} \int_{0}^{t} \int f^{\prime \prime}(x)\left(N\left(\sigma^{N}(x)\right)^{2}-\tilde{\sigma}^{2}(x)+H(x, x)-N H_{N}(x, x)\right) L_{N}(s)(d x) d s\right| \\
& \quad+\left|\frac{1}{2} \int_{0}^{t} \int f^{\prime \prime}(x)\left(\tilde{\sigma}^{2}(x)-H(x, x)\right)\left(L_{N}(s)(d x)-\mu_{s}(d x)\right) d s\right| \\
& \leq \frac{T}{2}\left\|f^{\prime \prime}\right\|_{L^{\infty}(\mathbb{R})}\left\|N\left(\sigma^{N}(x)\right)^{2}-\tilde{\sigma}^{2}(x)+H(x, x)-N H_{N}(x, x)\right\|_{L^{\infty}(\mathbb{R})} \\
& \quad+\frac{1}{2}\left|\int_{0}^{t}\left\langle f^{\prime \prime}(x)\left(\tilde{\sigma}^{2}(x)-H(x, x)\right), L_{N}(s)(d x)-\mu_{s}(d x)\right\rangle d s\right| \\
& \quad \longrightarrow 0,
\end{aligned}
$$

as $N \rightarrow \infty$, where the last step follows from the weak convergence of $\left\{L_{N}(t), t \in\right.$ $[0, T]\}_{N \in \mathbb{N}}$ and the continuity and boundedness of $f^{\prime \prime}(x)\left(\tilde{\sigma}^{2}(x)-H(x, x)\right)$ for $f \in \mathbb{F}$.

The fifth term on the right-hand side of (2.8) can be written as

$$
\begin{aligned}
& \frac{N}{2} \int_{0}^{t} \iint \frac{f^{\prime}(x)-f^{\prime}(y)}{x-y}\left[N H_{N}(x, y) L_{N}(s)(d x) L_{N}(s)(d y)-H(x, y) \mu_{s}(d x) \mu_{s}(d y)\right] d s \\
= & \frac{N}{2} \int_{0}^{t} \iint \frac{f^{\prime}(x)-f^{\prime}(y)}{x-y}\left[N H_{N}(x, y)-H(x, y)\right] L_{N}(s)(d x) L_{N}(s)(d y) d s \\
& +\frac{N}{2} \int_{0}^{t} \iint \frac{f^{\prime}(x)-f^{\prime}(y)}{x-y} H(x, y)\left[L_{N}(s)(d x)-\mu_{s}(d x)\right]\left[L_{N}(s)(d y)-\mu_{s}(d y)\right] d s \\
+ & \frac{N}{2} \int_{0}^{t} \iint \frac{f^{\prime}(x)-f^{\prime}(y)}{x-y} H(x, y) \mu_{s}(d x)\left[L_{N}(s)(d y)-\mu_{s}(d y)\right] d s \\
+ & \frac{N}{2} \int_{0}^{t} \iint \frac{f^{\prime}(x)-f^{\prime}(y)}{x-y} H(x, y)\left[L_{N}(s)(d x)-\mu_{s}(d x)\right] \mu_{s}(d y) d s \\
= & \frac{N}{2} \int_{0}^{t} \iint \frac{f^{\prime}(x)-f^{\prime}(y)}{x-y}\left[N H H_{N}(x, y)-H(x, y)\right] L_{N}(s)(d x) L_{N}(s)(d y) d s \\
& +\frac{N}{2} \int_{0}^{t} \iint \frac{f^{\prime}(x)-f^{\prime}(y)}{x-y} H(x, y)\left[L_{N}(s)(d x)-\mu_{s}(d x)\right]\left[L_{N}(s)(d y)-\mu_{s}(d y)\right] d s \\
& +\int_{0}^{t} \mathcal{L}_{s}^{N}\left(\int \frac{f^{\prime}(x)-f^{\prime}(y)}{x-y} H(x, y) \mu_{s}(d x)\right) d s,
\end{aligned}
$$

where the last equality follows from the symmetry of $\frac{f^{\prime}(x)-f^{\prime}(y)}{x-y} H(x, y)$. For the first term on the right-hand side of (2.11), by (2.2), we have

$$
\begin{aligned}
& \lim _{N \rightarrow \infty}\left|\frac{N}{2} \int_{0}^{t} \iint \frac{f^{\prime}(x)-f^{\prime}(y)}{x-y}\left[N H_{N}(x, y)-H(x, y)\right] L_{N}(s)(d x) L_{N}(s)(d y) d s\right| \\
\leq & \lim _{N \rightarrow \infty} \frac{N T}{2}\left\|\frac{f^{\prime}(x)-f^{\prime}(y)}{x-y}\right\|_{L^{\infty}\left(\mathbb{R}^{2}\right)}\left\|N H_{N}(x, y)-H(x, y)\right\|_{L^{\infty}\left(\mathbb{R}^{2}\right)}=0 .
\end{aligned}
$$

Therefore, by (1.10), (2.8) and the above estimations (2.9), (2.10), (2.11), and (2.12), 
we have that the term

$$
\begin{aligned}
& Q_{t}^{N}(f)-N M_{f}^{N}(t) \\
= & \mathcal{L}_{t}^{N}(f)-\mathcal{L}_{0}^{N}(f)-N M_{f}^{N}(t)-\int_{0}^{t} \mathcal{L}_{s}^{N}\left(f^{\prime} b\right) d s \\
- & \frac{1}{2} \int_{0}^{t}\left\langle f^{\prime \prime}(x)\left(\tilde{\sigma}^{2}(x)-H(x, x)\right), \mu_{s}(d x)\right\rangle d s \\
- & \int_{0}^{t} \mathcal{L}_{s}^{N}\left(\int \frac{f^{\prime}(x)-f^{\prime}(y)}{x-y} H(x, y) \mu_{s}(d x)\right) d s \\
- & \frac{N}{2} \int_{0}^{t} \iint \frac{f^{\prime}(x)-f^{\prime}(y)}{x-y} H(x, y)\left[L_{N}(s)(d x)-\mu_{s}(d x)\right]\left[L_{N}(s)(d y)-\mu_{s}(d y)\right] d s
\end{aligned}
$$

converges to 0 almost surely as $N \rightarrow \infty$, uniformly in $t \in[0, T]$. Note that in (2.9), (2.10) and (2.12), the integrand function is bounded, and hence the convergence is also in $L^{p}$ for all $p \geq 1$. Thus, $Q_{t}^{N}(f)-N M_{f}^{N}(t)$ with $f \in \mathbb{F}$ converges to 0 in $L^{p}$ for all $p \geq 1$ uniformly in $t \in[0, T]$.

Therefore, to prove the desired result, it suffices to show that, for any $k \in \mathbb{N}$ and $f_{1}, \ldots, f_{k} \in \mathbb{F}$, the vector-valued process $\left(N M_{f_{1}}^{N}(t), N M_{f_{2}}^{N}(t), \ldots, N M_{f_{k}}^{N}(t)\right)_{t \in[0, T]}$ converges in distribution to a centered Gaussian process $\left(G_{t}\left(f_{1}\right), G_{t}\left(f_{2}\right), \ldots, G_{t}\left(f_{k}\right)\right)_{t \in[0, T]}$ with covariance given by (2.3). To this end, by Lemma A.1 it suffices to prove that $\left\{N M_{f}^{N}(t), t \in[0, T]\right\}_{N \in \mathbb{N}}$ are martingales for $f \in \mathbb{F}$ such that the following limit holds in $L^{1}(\Omega)$,

$$
\lim _{N \rightarrow \infty}\left\langle N M_{f_{1}}^{N}, N M_{f_{2}}^{N}\right\rangle_{t}=\int_{0}^{t}\left\langle f_{1}^{\prime} f_{2}^{\prime} \tilde{\sigma}^{2}, \mu_{s}\right\rangle d s, \quad \forall f_{1}, f_{2} \in \mathbb{F} .
$$

By the uniform convergence of $\sqrt{N} \sigma^{N}$ towards $\tilde{\sigma}$, the boundedness of $f^{\prime}(x) \tilde{\sigma}(x)$ and (2.6), one can show that $\left\{N M_{f}^{N}(t), t \in[0, T]\right\}_{N \in \mathbb{N}}$ are martingales. It follows from (2.5) that, for $f_{1}, f_{2} \in \mathbb{F}$,

$$
\begin{aligned}
\left\langle N M_{f_{1}}^{N}, N M_{f_{2}}^{N}\right\rangle_{t} & =\sum_{i=1}^{N} \int_{0}^{t} f_{1}^{\prime}\left(x_{i}^{N}(s)\right) f_{2}^{\prime}\left(x_{i}^{N}(s)\right)\left(\sigma^{N}\left(x_{i}^{N}(s)\right)\right)^{2} d s \\
& =N \int_{0}^{t}\left\langle f_{1}^{\prime} f_{2}^{\prime}\left(\sigma^{N}\right)^{2}, L_{N}(s)\right\rangle d s \\
& =\int_{0}^{t}\left\langle f_{1}^{\prime} f_{2}^{\prime}\left(N\left(\sigma^{N}\right)^{2}-\tilde{\sigma}^{2}\right), L_{N}(s)\right\rangle d s+\int_{0}^{t}\left\langle f_{1}^{\prime} f_{2}^{\prime} \tilde{\sigma}^{2}, L_{N}(s)\right\rangle d s .
\end{aligned}
$$

The term $\int_{0}^{t}\left\langle f_{1}^{\prime} f_{2}^{\prime}\left(N\left(\sigma^{N}\right)^{2}-\tilde{\sigma}^{2}\right), L_{N}(s)\right\rangle d s$ converges to 0 a.s. and in $L^{p}$ for all $p \geq 1$ due to the boundedness of $f_{1}^{\prime}(x)$ and $f_{2}^{\prime}(x)$ and the uniform convergence of $\sqrt{N} \sigma^{N}$ towards $\tilde{\sigma}$. Furthermore, the following convergence

$$
\lim _{N \rightarrow \infty} \int_{0}^{t}\left\langle f_{1}^{\prime} f_{2}^{\prime} \tilde{\sigma}^{2}, L_{N}(s)\right\rangle d s=\int_{0}^{t}\left\langle f_{1}^{\prime} f_{2}^{\prime} \tilde{\sigma}^{2}, \mu_{s}\right\rangle d s,
$$

holds a.s. and in $L^{p}$ for all $p \geq 1$, because of the weak convergence of $\left\{L_{N}(t), t \in\right.$ $[0, T]\}_{N \in \mathbb{N}}$ to $\left\{\mu_{t}, t \in[0, T]\right\}$ and the boundedness of $f_{1}^{\prime} f_{2}^{\prime} \tilde{\sigma}^{2}$. Therefore, $\left\langle N M_{f_{1}}^{N}, N M_{f_{2}}^{N}\right\rangle_{t}$ converges to $\int_{0}^{t}\left\langle f_{1}^{\prime} f_{2}^{\prime} \tilde{\sigma}^{2}, \mu_{s}\right\rangle d s$ a.s. and in $L^{p}$ for all $p \geq 1$.

The proof is concluded.

If the particles in (1.6) are bounded, the test function space $\mathbb{F}$ can be enlarged by removing the boundedness condition in Theorem 2.2 . 
Corollary 2.3. Assume the same conditions as in Theorem 2.2. Moreover, for $T<\infty$, assume that

$$
\limsup _{N \rightarrow \infty} \sup _{t \in[0, T]} \max _{1 \leq i \leq N}\left|x_{i}^{N}(t)\right| \leq C(T)
$$

almost surely for some constant $C(T)$ depending on $T$. Then Theorem 2.2 still holds if the set $\mathbb{F}$ of test function is replaced by $C^{2}(\mathbb{R})$.

Proof. Note that by (2.14), all but finitely many terms in $\left\{\sup _{t \in[0, T]} \max _{1 \leq i \leq N}\left|x_{i}^{N}(t)\right|\right\}_{N \in \mathbb{N}}$ are bounded by $A(T)=C(T)+1$ a.s.. Thus, there exists a measurable set $A \subset \Omega$ with $\mathbb{P}(A)=1$ and a random variable $N_{0}(\omega) \in \mathbb{N}$, such that for $\omega \in A$, the empirical measures $\left\{L_{N}(t)(\omega), t \in[0, T]\right\}$ is supported in $[-A(T), A(T)]$ for all $N \geq N_{0}(\omega)$. Hence the limit $\left\{\mu_{t}, t \in[0, T]\right\}$ also has the same support. By (Rudin, 1991, 1.46), there exists a cut-off function $\eta(x) \in C^{\infty}(\mathbb{R})$ equal to 1 on $[-A(T), A(T)]$, of which the support is $[-2 A(T), 2 A(T)]$. If we replace $f$ by $f \eta$, noting that $f \eta \in \mathbb{F}$ for $f \in C^{2}(\mathbb{R})$ and that $f \eta=f$ on $[-A(T), A(T)]$, we can show that the term $Q_{t}^{N}(f)-N M_{f}^{N}(t)$ in (2.13) converges to 0 a.s. using the same argument as in the proof of Theorem 2.2. Then following the rest part of the proof, it is easy to get the result of Theorem 2.2.

Remark 2.4. Under the conditions in Theorem 2.2, (2.14) yields almost sure convergence of $Q_{t}^{N}(f)-N M_{f}^{N}(t)$ towards 0 for $f \in C^{2}(\mathbb{R})$. However, it is not clear whether the convergence also holds in $L^{p}$ for $p \geq 1$.

The following corollary provides a sufficient condition for $L^{p}$-convergence for $p \geq 1$.

Corollary 2.5. Assume the same conditions as in Theorem 2.2. For $T<\infty$ and all $p \geq 1$ and all $N \geq c p$ for some positive constant $c$, assume that

$$
\mathbb{E}\left[\sup _{t \in[0, T]}\left\langle|x|^{p}, L_{N}(t)\right\rangle\right] \leq C(T)^{p},
$$

for some positive constant $C(T)$ which depends only on $T$. Furthermore, assume that $\left(\tilde{\sigma}(x)^{2}-H(x, x)\right) f^{\prime \prime}(x)$ and its derivative has at most polynomial growth. Then for $f \in C^{3}(\mathbb{R})$ of which the derivatives have at most polynomial growth, $Q_{t}^{N}(f)-N M_{f}^{N}(t)$ converges to 0 in $L^{p}$ for all $p \geq 1$ uniformly in $t \in[0, T]$.

Proof. By the analysis in the proof of Theorem 2.2, it suffices to show

$$
\limsup _{N \rightarrow \infty} \mathbb{E}\left[\sup _{t \in[0, T]}\left|\left\langle g, L_{N}(t)\right\rangle-\left\langle g, \mu_{t}\right\rangle\right|^{p}\right]=0,
$$

for $p \geq 1$ and $g \in C^{1}(\mathbb{R})$ of which the derivative has at most polynomial growth. More precisely, one can check that under conditions (2.15) and (2.16), the convergences to 0 in (2.9), (2.10) and (2.12) are uniform in $L^{p}$, and hence $Q_{t}^{N}(f)-N M_{f}^{N}(t)$ in (2.13) converges to 0 in $L^{p}$ uniformly.

By Markov inequality and (2.15),

$$
\begin{aligned}
\mathbb{P}\left(\sup _{t \in[0, T]} \max _{1 \leq i \leq N}\left|x_{i}^{N}(t)\right|>C(T)+1\right) & \leq(C(T)+1)^{-p} \mathbb{E}\left[\sup _{t \in[0, T]} \max _{1 \leq i \leq N}\left|x_{i}^{N}(t)\right|^{p}\right] \\
& \leq(C(T)+1)^{-p} N \mathbb{E}\left[\sup _{t \in[0, T]}\left\langle|x|^{p}, L_{N}(t)\right\rangle\right] \\
& \leq N\left(\frac{C(T)}{C(T)+1}\right)^{p} .
\end{aligned}
$$


Choosing $p=\ln ^{2} N$, we have

$$
\begin{aligned}
\sum_{N=1}^{\infty} \mathbb{P}\left(\sup _{t \in[0, T]} \max _{1 \leq i \leq N}\left|x_{i}^{N}(t)\right|>C(T)+1\right) & \leq \sum_{N=1}^{\infty} N\left(\frac{C(T)}{C(T)+1}\right)^{p} \\
& =\sum_{N=1}^{\infty} N^{1+\ln N \ln \frac{C(T)}{C(T)+1}} \\
& <\infty
\end{aligned}
$$

By Borel-Cantelli lemma, we get that almost surely,

$$
\limsup _{N \rightarrow \infty} \sup _{t \in[0, T]} \max _{1 \leq i \leq N}\left|x_{i}^{N}(t)\right| \leq C(T)+1 .
$$

By the proof of Corollary 2.3, the limiting measure $\left\{\mu_{t}, t \in[0, T]\right\}$ is supported in $[-C(T)-1, C(T)+1]$.

For $g \in C^{1}(\mathbb{R})$ with $\left|g^{\prime}(x)\right| \leq a|x|^{n-1}+b$ for some $a, b \in \mathbb{R}, n \in \mathbb{N}_{+}$, define

$$
g_{\delta}(x)=g\left(\frac{x}{1+\delta x^{2}}\right)
$$

for $\delta>0$. Then $g_{\delta}(x)$ is a bounded continuous function, and hence

$$
\lim _{N \rightarrow \infty} \sup _{t \in[0, T]}\left|\left\langle g_{\delta}, L_{N}(t)\right\rangle-\left\langle g_{\delta}, \mu_{t}\right\rangle\right|=0,
$$

almost surely. By dominated convergence theorem,

$$
\lim _{N \rightarrow \infty} \mathbb{E}\left[\sup _{t \in[0, T]}\left|\left\langle g_{\delta}, L_{N}(t)\right\rangle-\left\langle g_{\delta}, \mu_{t}\right\rangle\right|^{p}\right]=0 .
$$

Note that $g^{\prime}(x)$ grows no faster than polynomials of degree $n-1$, by the mean value theorem, it is not difficult to show $\left|g(x)-g_{\delta}(x)\right| \leq C \delta\left(|x|^{n+2}+|x|^{3}\right)$, which implies that $g_{\delta}$ converges to $g$ uniformly in any compact interval as $\delta \rightarrow 0^{+}$. Thus,

$$
\lim _{\delta \rightarrow 0^{+}} \sup _{t \in[0, T]}\left|\left\langle g, \mu_{t}\right\rangle-\left\langle g_{\delta}, \mu_{t}\right\rangle\right|=0 .
$$

Finally, by Jensen's inequality and (2.15), we obtain that, as $\delta \rightarrow 0^{+}$,

$$
\begin{aligned}
& \mathbb{E}\left[\sup _{t \in[0, T]}\left|\left\langle g, L_{N}(t)\right\rangle-\left\langle g_{\delta}, L_{N}(t)\right\rangle\right|^{p}\right] \\
\leq & C^{p} \delta^{p} \mathbb{E}\left[\sup _{t \in[0, T]}\left|\left\langle|x|^{n+2}+|x|^{3}, L_{N}(t)\right\rangle\right|^{p}\right] \\
\leq & C^{p} \delta^{p} \mathbb{E}\left[\sup _{t \in[0, T]}\left|\left\langle\left(|x|^{n+2}+|x|^{3}\right)^{p}, L_{N}(t)\right\rangle\right|\right] \\
\leq & 2^{p} C^{p}\left(C(T)^{(n+2) p}+C(T)^{3 p}\right) \delta^{p} \rightarrow 0
\end{aligned}
$$

uniformly in $N \in \mathbb{N}_{+}$.

By (2.17), (2.18), (2.19) and the triangle inequality, we can obtain (2.16), and the proof is concluded.

Remark 2.6. The proof of Corollary 2.5 clearly shows that condition (2.15) implies condition (2.14). Therefore, under (2.15), for the test function $f$ satisfying conditions in Corollary 2.5, $Q_{t}^{N}(f)-N M_{f}^{N}(t)$ converges to 0 a.s. and in $L^{p}$ for all $p \geq 1$ uniformly in $t \in[0, T]$. As a consequence, Theorem 2.2 holds for such test functions. 
To end this section, we provide the following linear property for the Gaussian family $\left\{G_{t}(f), f \in \mathbb{F}\right\}$ obtained in Theorem 2.2.

Proposition 2.7. Consider the centered Gaussian family $\left\{G_{t}(f), f \in \mathbb{F}\right\}$ with covariance

$$
\mathbb{E}\left[G_{t}(f) G_{t}(g)\right]=\int_{0}^{t}\left\langle f^{\prime}(x) g^{\prime}(x) \tilde{\sigma}^{2}(x), \mu_{u}\right\rangle d u, \quad \forall f, g \in \mathbb{F} .
$$

For $f_{1}, f_{2} \in \mathbb{F}$ and $\alpha_{1}, \alpha_{2} \in \mathbb{R}$, we have

$$
G_{t}\left(\alpha_{1} f_{1}+\alpha_{2} f_{2}\right)=\alpha_{1} G_{t}\left(f_{1}\right)+\alpha_{2} G_{t}\left(f_{2}\right), \quad \forall t \in[0, T]
$$

almost surely.

Proof. For $f_{1}, f_{2} \in \mathbb{F}$ and $\alpha_{1}, \alpha_{2} \in \mathbb{R}$, it is easy to check that $\alpha_{1} f_{1}+\alpha_{2} f_{2} \in \mathbb{F}$. By the proof of Theorem 2.2, the random vector $\left(N M_{f_{1}}^{N}(t), N M_{f_{2}}^{N}(t), N M_{\alpha_{1} f_{1}+\alpha_{2} f_{2}}^{N}(t)\right)_{t \in[0, T]}$ converges in distribution to $\left(G_{t}\left(f_{1}\right), G_{t}\left(f_{2}\right), G_{t}\left(\alpha_{1} f_{1}+\alpha_{2} f_{2}\right)\right)_{t \in[0, T]}$. Hence, the linear combination $\left(\alpha_{1} N M_{f_{1}}^{N}(t)+\alpha_{2} N M_{f_{2}}^{N}(t)-N M_{\alpha_{1} f_{1}+\alpha_{2} f_{2}}^{N}(t)\right)_{t \in[0, T]}$ converges in distribution to $\left(\alpha_{1} G_{t}\left(f_{1}\right)+\alpha_{2} G_{t}\left(f_{2}\right)-G_{t}\left(\alpha_{1} f_{1}+\alpha_{2} f_{2}\right)\right)_{t \in[0, T]}$.

By (2.5), we can see that the martingale $M_{f}^{N}(t)$ is linear with respect to the function $f$, so $\alpha_{1} N M_{f_{1}}^{N}(t)+\alpha_{2} N M_{f_{2}}^{N}(t)=N M_{\alpha_{1} f_{1}+\alpha_{2} f_{2}}^{N}(t)$ for all $t \in[0, T]$ and all $N \in \mathbb{N}$, which implies that the process $\left(\alpha_{1} N M_{f_{1}}^{N}(t)+\alpha_{2} N M_{f_{2}}^{N}(t)-N M_{\alpha_{1} f_{1}+\alpha_{2} f_{2}}^{N}(t)\right)_{t \in[0, T]}$ is actually a zero process. Thus, as the limit of the convergence in distribution, $\left(\alpha_{1} G_{t}\left(f_{1}\right)+\alpha_{2} G_{t}\left(f_{2}\right)-\right.$ $\left.G_{t}\left(\alpha_{1} f_{1}+\alpha_{2} f_{2}\right)\right)_{t \in[0, T]}$ is also a zero process, which implies (2.20).

\section{Applications}

In this section, we first provide a comparison principle in Section 3.1, and then we apply our main results obtained in Section 2 to the eigenvalues of Wishart process (Section 3.2), Dyson's Brownian motion (Section 3.3) and the eigenvalues of symmetric matrix-valued Ornstein-Uhlenbeck process (Section 3.4).

For these three cases, we are able to obtain the boundedness of the moments for the empirical measures assuming proper initial conditions (see Lemma 3.4 for Wishart process, Eq. (3.30) for Dyson's Brownian motion, and Eq. (3.45) for Ornstein-Uhlenbeck process). This enables us to apply Corollary 2.5 and Remark 2.6. As a consequence, the CLT in Theorem 2.2 holds for a larger space of test functions consisting of all the functions in $C^{3}(\mathbb{R})$ of which the derivatives have at most polynomial growth. In particular, if we choose the space $\mathbb{R}[x]$ of polynomial functions as the space of test functions, we are able to obtain recursive formulas for the basis $\left\{\mathcal{L}_{t}\left(x^{n}\right), t \in[0, T]\right\}_{n \in \mathbb{N}}$ of $\left\{\mathcal{L}_{t}(f), t \in[0, T]\right\}_{f \in \mathbb{R}[x]}$. Note that these results are more precise than the general results in Section 2, where we study the centered processes $\left\{Q_{t}^{N}(f)\right\}_{N \in \mathbb{N}}$ for more restricted test functions $f \in \mathbb{F} \subset C_{b}^{2}(\mathbb{R})$, recalling that $\mathbb{F}$ is given in (2.1).

\subsection{Comparison principle}

In this subsection, we provide comparison principles for SDE (1.2) and particle system (1.6), which allow us to obtain the boundedness of the eigenvalues/particles under more general initial conditions in Sections 3.2, 3.3 and 3.4.

Throughout this subsection, the dimension $N$ is fixed and thus subscripts/superscripts are removed. Precisely, consider the following two particle systems: for $1 \leq i \leq N, t \geq 0$,

$$
\left\{\begin{array}{l}
d x_{i}(t)=\sigma_{i}\left(x_{i}(t)\right) d W_{i}(t)+\left(b_{i}\left(x_{i}(t)\right)+\sum_{j: j \neq i} \frac{H_{i j}\left(x_{i}(t), x_{j}(t)\right)}{x_{i}(t)-x_{j}(t)}\right) d t \\
x_{1}(t) \leq \ldots \leq x_{N}(t)
\end{array}\right.
$$


and

$$
\left\{\begin{array}{l}
d y_{i}(t)=\sigma_{i}\left(y_{i}(t)\right) d W_{i}(t)+\left(\tilde{b}_{i}\left(y_{i}(t)\right)+\sum_{j: j \neq i} \frac{H_{i j}\left(y_{i}(t), y_{j}(t)\right)}{y_{i}(t)-y_{j}(t)}\right) d t \\
y_{1}(t) \leq \ldots \leq y_{N}(t)
\end{array}\right.
$$

with non-colliding initial values $x(0)=\left(x_{1}(0), \ldots, x_{N}(0)\right)$ and $y(0)=\left(y_{1}(0), \ldots, y_{N}(0)\right)$, respectively. Here, the functions $\sigma_{i}(x), b_{i}(x)$ and $\tilde{b}_{i}(x)$ for $1 \leq i \leq N$ are continuous, and $H_{i j}(x, y)$ with $i \neq j$ is a continuous, non-negative and symmetric function satisfying the condition (Graczyk and Małecki, 2014, (A1)):

$$
\frac{H_{i j}(w, z)}{z-w} \leq \frac{H_{i j}(x, y)}{y-x}, \quad \forall w<x<y<z, \quad 1 \leq i \neq j \leq N
$$

Note that conditions for the existence and uniqueness of a non-colliding and nonexploding strong solution to (3.1) (or (3.2)) were obtained in Graczyk and Małecki (2014). In particular, under conditions (A2) - (A5) therein, the particles will separate from each other immediately after starting from a colliding initial state, and will not collide forever.

Theorem 3.1. Suppose $x(t)=\left(x_{1}(t), \ldots, x_{N}(t)\right)$ and $y(t)=\left(y_{1}(t), \ldots, y_{N}(t)\right)$ are the non-exploding and non-colliding unique strong solutions to (3.1) and (3.2), respectively. Assume that there exists a strictly increasing function $\rho:[0, \infty) \rightarrow[0, \infty)$ with $\rho(0)=0$ and

$$
\int_{0+} \rho^{-2}(u) d u=\infty
$$

such that

$$
\left|\sigma_{i}(u)-\sigma_{i}(v)\right| \leq \rho(|u-v|), \quad \forall u, v \in \mathbb{R}, 1 \leq i \leq N .
$$

If we further assume that $b_{i}(u) \leq \tilde{b}_{i}(u)$ for all $u \in \mathbb{R}$, and $x_{i}(0) \leq y_{i}(0)$ a.s., $1 \leq i \leq N$, then

$$
\mathbb{P}\left(x_{i}(t) \leq y_{i}(t), \forall t \geq 0,1 \leq i \leq N\right)=1 \text {. }
$$

Proof. The continuity of the functions $H_{i j}$ and the condition (3.3) imply that for all $1 \leq i \neq j \leq N$,

$$
\frac{H_{i j}(x, z)}{x-z} \geq \frac{H_{i j}(x, y)}{x-y}, \quad \forall x<y \leq z
$$

and

$$
\frac{H_{i j}(w, y)}{y-w} \leq \frac{H_{i j}(x, y)}{y-x}, \quad \forall w \leq x<y
$$

Hence, the drift functions

$$
F(u)=\left(b_{i}\left(u_{i}\right)+\sum_{j: j \neq i} \frac{H_{i j}\left(u_{i}, u_{j}\right)}{u_{i}-u_{j}}\right)_{1 \leq i \leq N}, \quad \widetilde{F}(u)=\left(\tilde{b}_{i}\left(u_{i}\right)+\sum_{j: j \neq i} \frac{H_{i j}\left(u_{i}, u_{j}\right)}{u_{i}-u_{j}}\right)_{1 \leq i \leq N},
$$

satisfy the quasi-monotonously increasing condition in Lemma A.2.

In order to apply Lemma A.2 to get the desired result, we use an approximation argument to remove the singularities of the drift functions $F$ and $\widetilde{F}$. For $\epsilon>0$, let

$$
\Delta_{\epsilon}=\left\{u=\left(u_{1}, \ldots, u_{N}\right) \in \mathbb{R}^{N}: \forall 1 \leq i \leq N-1, u_{i+1}-u_{i}>\epsilon\right\}
$$


and define the stopping time

$$
\tau_{\epsilon}=\inf _{t>0}\left\{\min _{1 \leq i \leq N-1}\left(x_{i+1}(t)-x_{i}(t)\right) \wedge\left(y_{i+1}(t)-y_{i}(t)\right) \leq \epsilon\right\} .
$$

One can find continuous quasi-monotonously increasing functions $F_{\epsilon}$ and $\widetilde{F}_{\epsilon}$, such that they coincide with $F$ and $\widetilde{F}$ in $\Delta_{\epsilon}$, respectively. Before time $\tau_{\epsilon}$, both $x$-particles and $y$-particles stay in $\Delta_{\epsilon}$ and thus satisfy (3.1) and (3.2) with drift functions $\widetilde{F}_{\epsilon}$ and $\widetilde{F}_{\epsilon}$, respectively.

Applying Lemma A.2 to the processes $x^{\epsilon}$ and $y^{\epsilon}$, we have

$$
\mathbb{P}\left(x_{i}^{\epsilon}(t) \leq y_{i}^{\epsilon}(t), \forall t \geq 0,1 \leq i \leq N\right)=1,
$$

which implies

$$
\mathbb{P}\left(x_{i}(t) \leq y_{i}(t), \forall t \in\left[0, \tau_{\epsilon}\right], 1 \leq i \leq N\right)=1 .
$$

The desired result now follows from the non-colliding property $\lim _{\epsilon \rightarrow 0^{+}} \tau_{\epsilon}=\infty$.

As a corollary of Theorem 3.1, we have the following comparison principle for SDE (1.2) of eigenvalue processes. Note that the existence and uniqueness of the non-colliding and non-exploding strong solution was obtained under proper conditions in Graczyk and Małecki (2013).

Corollary 3.2. Suppose that the following systems of eigenvalue SDEs

$d \lambda_{i}(t)=2 g_{N}\left(\lambda_{i}(t)\right) h_{N}\left(\lambda_{i}^{N}(t)\right) d W_{i}(t)+\left(b_{N}\left(\lambda_{i}^{N}(t)\right)+\sum_{j: j \neq i} \frac{G_{N}\left(\lambda_{i}(t), \lambda_{j}(t)\right)}{\lambda_{i}(t)-\lambda_{j}(t)}\right) d t, 1 \leq i \leq N$, $\lambda_{1}(t) \leq \ldots \leq \lambda_{N}(t), t \geq 0$,

and

$d \theta_{i}(t)=2 g_{N}\left(\theta_{i}(t)\right) h_{N}\left(\theta_{i}^{N}(t)\right) d W_{i}(t)+\left(\tilde{b}_{N}\left(\theta_{i}^{N}(t)\right)+\sum_{j: j \neq i} \frac{G_{N}\left(\theta_{i}(t), \theta_{j}(t)\right)}{\theta_{i}(t)-\theta_{j}(t)}\right) d t, 1 \leq i \leq N$,

$\theta_{1}(t) \leq \ldots \leq \theta_{N}(t), t \geq 0$

with non-colliding initial values $\lambda(0)=\left(\lambda_{1}(0), \ldots, \lambda_{N}(0)\right)$ and $\theta(0)=\left(\theta_{1}(0), \ldots, \theta_{N}(0)\right)$, respectively, have non-exploding and non-colliding unique strong solutions $\lambda(t)=$ $\left(\lambda_{1}(t), \ldots, \lambda_{N}(t)\right)$ and $\theta(t)=\left(\theta_{1}(t), \ldots, \theta_{N}(t)\right)$, respectively. Here, $g_{N}(x), h_{N}(x), b_{N}(x)$ and $\tilde{b}_{N}(x)$ are continuous functions, and $G_{N}(x, y)=g_{N}^{2}(x) h_{N}^{2}(y)+g_{N}^{2}(y) h_{N}^{2}(x)$ satisfies

$$
\frac{G_{N}(w, z)}{z-w} \leq \frac{G_{N}(x, y)}{y-x}, \quad \forall w<x<y<z .
$$

Assume that there exists a strictly increasing function $\rho:[0, \infty) \rightarrow[0, \infty)$ with $\rho(0)=0$ and

$$
\int_{0^{+}} \rho^{-2}(u) d u=\infty
$$

such that

$$
\left|g_{N}(u) h_{N}(u)-g_{N}(v) h_{N}(v)\right| \leq \rho(|u-v|), \quad \forall u, v \in \mathbb{R} .
$$

Furthermore, we assume that $b_{N}(u) \leq \tilde{b}_{N}(u)$ for all $u \in \mathbb{R}$. If $\lambda_{i}(0) \leq \theta_{i}(0)$ for all $1 \leq i \leq N$ almost surely, then

$$
\mathbb{P}\left(\lambda_{i}(t) \leq \theta_{i}(t), \forall t \geq 0,1 \leq i \leq N\right)=1 .
$$




\subsection{Application to eigenvalues of Wishart process}

In this subsection, we discuss the limit theorem for Wishart process. As illustrated in Graczyk and Małecki (2013) and Song et al. (2020), the scaled Wishart process $X_{t}^{N}=\tilde{B}^{\top}(t) \tilde{B}(t) / N$, where $\tilde{B}(t)$ is a $P \times N$ matrix-valued Brownian motion with $P>N-1$, is the solution to (1.1) with the coefficient functions

$$
g_{N}(x) h_{N}(y)=\frac{\sqrt{x}}{\sqrt{N}}, \quad b_{N}(x)=\frac{P}{N} .
$$

The eigenvalue processes now satisfy

$$
d \lambda_{i}^{N}(t)=2 \frac{\sqrt{\lambda_{i}^{N}(t)}}{\sqrt{N}} d W_{i}(t)+\left(\frac{P}{N}+\frac{1}{N} \sum_{j: j \neq i} \frac{\lambda_{i}^{N}(t)+\lambda_{j}^{N}(t)}{\lambda_{i}^{N}(t)-\lambda_{j}^{N}(t)}\right) d t, 1 \leq i \leq N, t \geq 0 .
$$

Hence, the eigenvalue processes are the particles in (1.6) with

$$
\sigma^{N}(x)=2 \frac{\sqrt{x}}{\sqrt{N}}, b_{N}(x)=\frac{P}{N}, H_{N}(x, y)=\frac{x+y}{N},
$$

and thus,

$$
N H_{N}(x, y)=H(x, y)=x+y, b(x)=\lim _{N \rightarrow \infty} \frac{P}{N}=c \geq 1, \sqrt{N} \sigma^{N}(x)=\tilde{\sigma}(x)=2 \sqrt{x} .
$$

By (Graczyk and Małecki, 2019, Theorem 3), all the components of the solution to (3.5) are non-negative if all the components of the initial value are non-negative. Let $\mathbb{P}^{N}$ be the distribution on $\Delta_{N}=\left\{x=\left(x_{1}, x_{2}, \ldots, x_{N}\right) \in \mathbb{R}^{N}: 0<x_{1}<\ldots<x_{N}\right\}$ with density

$$
p(x)=C_{N, P} \exp \left(-\frac{N}{2} \sum_{i=1}^{N} x_{i}\right) \prod_{i=1}^{N} x_{i}^{(P-N-1) / 2} \prod_{1 \leq j<i \leq N}\left(x_{i}-x_{j}\right)
$$

where $C_{N, P}>0$ is a normalization constant. Then we have the following estimation on the eigenvalues.

Lemma 3.3. Let $\xi^{N}=\left(\xi_{1}^{N}, \ldots, \xi_{N}^{N}\right)$ be a random vector independent of $\left(W_{1}, \ldots, W_{N}\right)$ with (3.7) as its joint probability density function. Assume that $\left(\lambda_{1}^{N}(0), \ldots, \lambda_{N}^{N}(0)\right)$ is independent of $\left(W_{1}, \ldots, W_{N}\right)$ and that there exists a constant $a>0$, such that $\lambda_{i}^{N}(0) \leq$ $a \xi_{i}^{N}$ for $1 \leq i \leq N$ almost surely. Then there exists a stationary stochastic process $u^{N}(t)$ with initial value $u^{N}(0)=\xi^{N}$ satisfying, for $1 \leq i \leq N$ and $t \geq 0$,

$$
\lambda_{i}^{N}(t) \leq v_{i}^{N}(t)=(t+a) u_{i}^{N}(t) .
$$

Proof. Consider the following system of SDEs, for $1 \leq i \leq N$,

$$
d u_{i}^{N}(t)=2 \frac{\sqrt{u_{i}^{N}(t)}}{\sqrt{N(t+a)}} d W_{i}(t)+\frac{1}{t+a}\left(\frac{P}{N}-u_{i}^{N}(t)+\frac{1}{N} \sum_{j: j \neq i} \frac{u_{i}^{N}(t)+u_{j}^{N}(t)}{u_{i}^{N}(t)-u_{j}^{N}(t)}\right) d t, \quad t \geq 0
$$

with initial value $u_{i}^{N}(0)=\xi_{i}^{N}(0)$ distributed according to $\mathbb{P}^{N}$ and $u_{1}^{N}(t) \leq \ldots \leq u_{N}^{N}(t)$.

Note that the pathwise uniqueness proved in (Graczyk and Małecki, 2013, Theorem 2 ) is still valid if the coefficient functions depend on the time $t$ and the corresponding conditions therein hold uniformly in $t$. Furthermore, the boundedness estimation and the McKean's argument in (Graczyk and Małecki, 2013, Theorem 5) is also valid when $t \geq 0$. Therefore, the system of SDEs (3.8) has a unique non-colliding strong solution. 
If at any time $t, u^{N}(t)$ has the distribution $\mathbb{P}^{N}$, then Lemma A.3 yields that $\frac{d}{d t} \mathbb{E}\left[f\left(u^{N}(t)\right)\right]$ vanishes for $f \in C_{b}^{2}(\mathbb{R})$. Since $u^{N}(0)$ is distributed according to $\mathbb{P}^{N}$, we can conclude that $\left(u^{N}(t)\right)_{t \geq 0}$ is a stationary process with marginal distribution $\mathbb{P}^{N}$.

Now let $v_{i}^{N}(t)=(t+a) u_{i}^{N}(t)$ for $1 \leq i \leq N$ and $v^{N}(t)=\left(v_{1}^{N}(t), \ldots, v_{N}^{N}(t)\right)$. Then the Itô formula shows that $v^{N}(t)$ is a solution to (3.5) with initial value $v^{N}(0)=a u^{N}(0)=a \xi^{N}$. Noting that the solution of (3.5) is non-negative and that $G_{N}(x, y)=(x+y) / N$ with non-negative variables satisfies condition (3.3), we can apply the comparison principle in Theorem 3.1 to obtain

$$
\lambda_{i}^{N}(t) \leq v_{i}^{N}(t)=(t+a) u_{i}^{N}(t)
$$

The proof is concluded.

Lemma 3.4. Assume the same conditions as in Lemma 3.3. Then for any $T<\infty$, there exists a positive constant $C(a, T)$ depending only on $(a, T)$, such that for all $p \geq 1$,

$$
\mathbb{E}\left[\sup _{t \in[0, T]}\left\langle|x|^{p}, L_{N}(t)\right\rangle\right] \leq C(a, T)^{p},
$$

almost surely for $N \geq(2 p-1) / \alpha$ for some positive constant $\alpha$.

Proof. Noting that the probability density of $u^{N}(t)$ considered in Lemma 3.3 is (3.7) for all $t$, we can obtain the following tail probability estimation with $\alpha$ being a positive constant independent of $N$,

$$
\mathbb{P}\left(u_{N}^{N}(t) \geq x\right)=\mathbb{P}^{N}\left(x_{N} \geq x\right) \leq \exp (-\alpha N x), \text { for } t \geq 0 .
$$

By Lemma 3.3 and (3.9), we have for $t \geq 0$,

$$
\begin{aligned}
\mathbb{E}\left[\lambda_{N}^{N}(t)^{k}\right] & \leq(t+a)^{k} \mathbb{E}\left[u_{N}^{N}(t)^{k}\right]=k(t+a)^{k} \int_{0}^{\infty} x^{k-1} \mathbb{P}\left(u_{N}^{N}(t) \geq x\right) d x \\
& \leq k(t+a)^{k} \int_{0}^{\infty} x^{k-1} \exp (-\alpha N x) d x=\frac{\Gamma(k+1)}{(\alpha N)^{k}}(t+a)^{k} \\
& \leq(t+a)^{k}
\end{aligned}
$$

for $k \in[0, \alpha N]$, where $\Gamma(x)$ is the gamma function.

Now we apply (2.4) and (2.6) with $f(x)=x^{n+2}$ for $n \geq-1$ to obtain

$$
\begin{aligned}
\left\langle x^{n+2}, L_{N}(t)\right\rangle= & \left\langle x^{n+2}, L_{N}(0)\right\rangle+M_{x^{n+2}}^{N}(t)+\frac{(P+n+1)(n+2)}{N} \int_{0}^{t}\left\langle x^{n+1}, L_{N}(s)\right\rangle d s \\
& +\frac{n+2}{2} \int_{0}^{t} \iint \sum_{k=0}^{n} x^{k} y^{n-k}(x+y) L_{N}(s)(d x) L_{N}(s)(d y) d s .
\end{aligned}
$$

where the martingale term $M_{x^{n+2}}^{N}(t)$ has the quadratic variation

$$
\left\langle M_{x^{n+2}}^{N}\right\rangle_{t}=\frac{4(n+2)^{2}}{N^{2}} \int_{0}^{t}\left\langle x^{2 n+3}, L_{N}(s)\right\rangle d s .
$$

By the Cauchy-Schwarz inequality, Burkholder-Davis-Gundy inequality, Hölder inequality and the estimation (3.10), for $(2 n+3) q \leq \alpha N, q \in \mathbb{N}$, and $\Lambda_{q}$ being a positive constant 
depending only on $q$,

$$
\begin{aligned}
& \mathbb{E}\left[\left|\sup _{u \in[0, t]} M_{x^{n+2}}^{N}(u)\right|^{q}\right] \leq\left\{\mathbb{E}\left[\sup _{u \in[0, t]} M_{x^{n+2}}^{N}(u)^{2 q}\right]\right\}^{1 / 2} \\
\leq & \sqrt{\Lambda_{q}}\left\{\mathbb{E}\left[\left\langle M_{x^{n+2}}^{N}\right\rangle_{t}^{q}\right]\right\}^{1 / 2} \leq \frac{2^{q}(n+2)^{q} \sqrt{\Lambda_{q}}}{N^{q}}\left\{\mathbb{E}\left[\int_{0}^{t}\left\langle x^{2 n+3}, L_{N}(s)\right\rangle d s\right]^{q}\right\}^{1 / 2} \\
= & \frac{2^{q}(n+2)^{q} \sqrt{\Lambda_{q}}}{N^{q}}\left\{\mathbb{E}\left[\int_{0}^{t} \frac{1}{N} \sum_{i=1}^{N} \lambda_{i}^{N}(s)^{2 n+3} d s\right]^{q}\right\}^{1 / 2} \\
\leq & \frac{2^{q}(n+2)^{q} \sqrt{\Lambda_{q}}}{N^{q}}\left\{\mathbb{E}\left[\int_{0}^{t} \lambda_{N}^{N}(s)^{2 n+3} d s\right]^{q}\right\}^{1 / 2} \\
\leq & \frac{2^{q}(n+2)^{q} \sqrt{\Lambda_{q}}}{N^{q}}\left\{\mathbb{E}\left[t^{q-1} \int_{0}^{t} \lambda_{N}^{N}(s)^{(2 n+3) q} d s\right]\right\}^{1 / 2} \\
\leq & \frac{2^{q}(n+2)^{q} \sqrt{\Lambda_{q}}}{N^{q}}\left\{t^{q-1} \int_{0}^{t}(s+a)^{(2 n+3) q} d s\right\}^{1 / 2} \\
\leq & \frac{2^{q}(n+2)^{q} \sqrt{\Lambda_{q} t^{q}(t+a)^{(2 n+3) q}}}{N^{q}} .
\end{aligned}
$$

Defining, for $k \geq 1$,

$$
E_{t}^{N}(k)=\mathbb{E}\left[\sup _{u \in[0, t]}\left\langle x^{k}, L_{N}(u)\right\rangle\right],
$$

it follows from (3.11) that for $n \geq-1$,

$$
\begin{aligned}
& E_{t}^{N}(n+2) \\
& \leq E_{0}^{N}(n+2)+\mathbb{E}\left[\sup _{u \in[0, t]} M_{x^{n+2}}^{N}(u)\right]+\frac{(P+n+1)(n+2)}{N} \mathbb{E}\left[\sup _{u \in[0, t]} \int_{0}^{u}\left\langle x^{n+1}, L_{N}(s)\right\rangle d s\right] \\
& \quad+\frac{n+2}{2} \mathbb{E}\left[\sup _{u \in[0, t]} \int_{0}^{u} \iint \sum_{k=0}^{n} x^{k} y^{n-k}(x+y) L_{N}(s)(d x) L_{N}(s)(d y) d s\right] .
\end{aligned}
$$

For the third and the fourth terms on the right-hand side of (3.13), we have by (3.10),

$$
\begin{aligned}
& \frac{(P+n+1)(n+2)}{N} \mathbb{E}\left[\sup _{u \in[0, t]} \int_{0}^{u}\left\langle x^{n+1}, L_{N}(s)\right\rangle d s\right] \\
\leq & \frac{(P+n+1)(n+2)}{N} \mathbb{E}\left[\int_{0}^{t}\left|\lambda_{N}^{N}(s)\right|^{n+1} d s\right] \\
\leq & \frac{(P+n+1)(n+2)}{N} \int_{0}^{t}(s+a)^{n+1} d s \\
\leq & \frac{(P+n+1)(n+2) t(t+a)^{n+1}}{N},
\end{aligned}
$$


and

$$
\begin{aligned}
& \frac{n+2}{2} \mathbb{E}\left[\sup _{u \in[0, t]} \int_{0}^{u} \iint \sum_{k=0}^{n} x^{k} y^{n-k}(x+y) L_{N}(s)(d x) L_{N}(s)(d y) d s\right] \\
= & \frac{n+2}{2} \sum_{k=0}^{n} \mathbb{E}\left[\sup _{u \in[0, t]} \int_{0}^{u}\left(\left\langle x^{k+1}, L_{N}(s)\right\rangle\left\langle y^{n-k}, L_{N}(s)\right\rangle+\left\langle x^{k}, L_{N}(s)\right\rangle\left\langle y^{n+1-k}, L_{N}(s)\right\rangle\right) d s\right] \\
\leq & \frac{(n+2)}{2} \sum_{k=0}^{n} \mathbb{E}\left[\sup _{u \in[0, t]} \int_{0}^{u}\left(\left|\lambda_{N}^{N}(s)\right|^{k+1}\left|\lambda_{N}^{N}(s)\right|^{n-k}+\left|\lambda_{N}^{N}(s)\right|^{k}\left|\lambda_{N}^{N}(s)\right|^{n+1-k}\right) d s\right] \\
\leq & (n+2)(n+1) \mathbb{E}\left[\int_{0}^{t}\left|\lambda_{N}^{N}(s)\right|^{n+1} d s\right] \\
\leq & (n+2)(n+1) t(t+a)^{n+1}
\end{aligned}
$$

for $n+1 \leq \alpha N$. Hence, by (3.12), (3.13), and the above two estimations, for $n \geq-1$ such that $2 n+3 \leq \alpha N$ and $t \in[0, T]$, we have

$$
\begin{aligned}
E_{t}^{N}(n+2) \leq & E_{0}^{N}(n+2)+\frac{2(n+2) \sqrt{\Lambda_{1} t(t+a)^{2 n+3}}}{N} \\
& +\frac{(P+n+1)(n+2) t(t+a)^{n+1}}{N}+(n+2)(n+1) t(t+a)^{n+1}
\end{aligned}
$$

Thus, for all $-1 \leq n \leq \frac{\alpha N-3}{2}$, noting that $E_{0}^{N}(n+2) \leq \mathbb{E}\left[\lambda_{N}^{N}(0)^{n+2}\right] \leq a^{n+2}$ by (3.10), we have

$$
E_{T}^{N}(n+2) \leq C_{a, T}^{n+2}
$$

for some positive constant $C_{a, T}$ depending on $(a, T)$ only.

The proof is concluded.

Under the conditions in Lemma 3.3, Lemma 3.4 implies $\lim \sup _{N \rightarrow \infty} \sup _{N} \lambda_{N}^{N}(0)<\infty$ almost surely. One can check that the conditions (A) - (D) in Song et al. (2020) are satisfied, hence $\left\{L_{N}(t), t \in[0, T]\right\}_{n \in \mathbb{N}}$ is tight (see also (Song et al., 2020, Remark 3.3)), and we know that it converges to $\left\{\mu_{t}, t \in[0, T]\right\}$, where $\mu_{t}$ is a scaled Marchenko-Pastur law. Recall that $c=\lim _{N \rightarrow \infty} P / N$ and that $\mathcal{L}_{t}^{N}(f)$ is defined by (1.9) in Theorem 2.2.

Theorem 3.5. Assume that $\lim _{N \rightarrow \infty}|P-c N|=0$, and that for any polynomial $f(x) \in$ $\mathbb{R}[x]$, the initial value $\mathcal{L}_{0}^{N}(f)$ converges in probability to a random variable $\mathcal{L}_{0}(f)$. Besides, assume the same condition on $\left\{\lambda_{i}^{N}(0), i=1,2, \ldots, N\right\}$ as in Lemma 3.3 for all $N \in \mathbb{N}$. Furthermore, assume that for all $n \in \mathbb{N}$,

$$
\sup _{N} \mathbb{E}\left[\left|N\left(\left\langle x^{n}, L_{N}(0)\right\rangle-\left\langle x^{n}, \mu_{0}\right\rangle\right)\right|^{q}\right]<\infty
$$

for all $q \geq 1$. Then for any $0<T<\infty$, there exists a family of processes $\left\{\mathcal{L}_{t}(f), t \in\right.$ $[0, T]\}_{f \in \mathbb{R}[x]}$, such that for any $n \in \mathbb{N}$ and any polynomials $P_{1}, \ldots, P_{n} \in \mathbb{R}[x]$, the vectorvalued process $\left(\mathcal{L}_{t}^{N}\left(P_{1}\right), \ldots, \mathcal{L}_{t}^{N}\left(P_{n}\right)\right)_{t \in[0, T]}$ converges to $\left(\mathcal{L}_{t}\left(P_{1}\right), \ldots, \mathcal{L}_{t}\left(P_{n}\right)\right)_{t \in[0, T]}$ in distribution, as $N \rightarrow \infty$.

The limit processes $\left\{\mathcal{L}_{t}(f), t \in[0, T]\right\}_{f \in \mathbb{R}[x]}$ are characterized by the following properties.

1. For $P_{1}, P_{2} \in \mathbb{R}[x], \alpha_{1}, \alpha_{2} \in \mathbb{R}, t \in[0, T]$,

$$
\mathcal{L}_{t}\left(\alpha_{1} P_{1}+\alpha_{2} P_{2}\right)=\alpha_{1} \mathcal{L}_{t}\left(P_{1}\right)+\alpha_{2} \mathcal{L}_{t}\left(P_{2}\right) .
$$


2. The basis $\left\{\mathcal{L}_{t}\left(x^{n}\right), t \in[0, T]\right\}_{n \in \mathbb{N}}$ of $\left\{\mathcal{L}_{t}(P), t \in[0, T]\right\}_{P \in \mathbb{R}[x]}$ satisfies

$$
\mathcal{L}_{t}(1)=0, \quad \mathcal{L}_{t}(x)=\mathcal{L}_{0}(x)+G_{t}(x)
$$

and for $n \geq 0$,

$$
\begin{aligned}
\mathcal{L}_{t}\left(x^{n+2}\right) & =\mathcal{L}_{0}\left(x^{n+2}\right)+c(n+2) \int_{0}^{t} \mathcal{L}_{s}\left(x^{n+1}\right) d s+(n+2)(n+1) \int_{0}^{t}\left\langle x^{n+1}, \mu_{s}\right\rangle d s \\
& +(n+2) \sum_{k=0}^{n} \int_{0}^{t}\left(\mathcal{L}_{s}\left(x^{n-k}\right)\left\langle x^{k+1}, \mu_{s}\right\rangle+\mathcal{L}_{s}\left(x^{n+1-k}\right)\left\langle x^{k}, \mu_{s}\right\rangle\right) d s+G_{t}\left(x^{n+2}\right),
\end{aligned}
$$

where $\left\{G_{t}\left(x^{n}\right), t \in[0, T]\right\}_{n \in \mathbb{N}}$ is a family of centered Gaussian processes with covariance

$$
\mathbb{E}\left[G_{t}\left(x^{n}\right) G_{s}\left(x^{m}\right)\right]=4 m n \int_{0}^{t \wedge s}\left\langle x^{n+m-1}, \mu_{u}\right\rangle d u, \quad n, m \geq 1
$$

Proof. First, note that by Lemma 3.4 and Corollary 2.5, $Q_{t}^{N}\left(x^{n}\right)$ defined by (1.10) converges in distribution to a centered Gaussian family $\left\{G_{t}\left(x^{n}\right), t \in[0, T]\right\}_{n \in \mathbb{N}}$ with covariance given by (3.16). Furthermore, by (1.9), (1.10) and (3.6), for $n \geq-1$, we have

$$
\begin{aligned}
& Q_{t}^{N}\left(x^{n+2}\right) \\
&=\mathcal{L}_{t}^{N}\left(x^{n+2}\right)-\mathcal{L}_{0}^{N}\left(x^{n+2}\right)-c(n+2) \int_{0}^{t} \mathcal{L}_{s}^{N}\left(x^{n+1}\right) d s-(n+2)(n+1) \int_{0}^{t}\left\langle x^{n+1}, \mu_{s}\right\rangle d s \\
& \quad-(n+2) \int_{0}^{t} \mathcal{L}_{s}^{N}\left(\int \sum_{k=0}^{n} x^{k} y^{n-k}(x+y) \mu_{s}(d x)\right) d s \\
& \quad-\frac{N(n+2)}{2} \int_{0}^{t} \iint \sum_{k=0}^{n} x^{k} y^{n-k}(x+y)\left[L_{N}(s)(d x)-\mu_{s}(d x)\right]\left[L_{N}(s)(d y)-\mu_{s}(d y)\right] d s \\
&=\mathcal{L}_{t}^{N}\left(x^{n+2}\right)-\mathcal{L}_{0}^{N}\left(x^{n+2}\right)-c(n+2) \int_{0}^{t} \mathcal{L}_{s}^{N}\left(x^{n+1}\right) d s-(n+2)(n+1) \int_{0}^{t}\left\langle x^{n+1}, \mu_{s}\right\rangle d s \\
& \quad-(n+2) \sum_{k=0}^{n} \int_{0}^{t}\left(\mathcal{L}_{s}^{N}\left(x^{n-k}\right)\left\langle x^{k+1}, \mu_{s}\right\rangle+\mathcal{L}_{s}^{N}\left(x^{n+1-k}\right)\left\langle x^{k}, \mu_{s}\right\rangle\right) d s \\
& \quad-\frac{(n+2)}{2 N} \sum_{k=0}^{n} \int_{0}^{t}\left(\mathcal{L}_{s}^{N}\left(x^{n-k}\right) \mathcal{L}_{s}^{N}\left(x^{k+1}\right)+\mathcal{L}_{s}^{N}\left(x^{n+1-k}\right) \mathcal{L}_{s}^{N}\left(x^{k}\right)\right) d s .
\end{aligned}
$$

In Corollary 2.3 and Corollary 2.5, we have shown $Q_{t}^{N}\left(x^{n+2}\right)-N M_{x^{n+2}}^{N}$ converges to 0 almost surely and in $L^{q}$ for all $q \geq 1$ as $N \rightarrow \infty$, uniformly in $t \in[0, T]$. Thus, by (3.12), (3.17), and the condition (3.14), it is not difficult to show

$$
\sup _{N \in \mathbb{N}} \mathbb{E}\left[\sup _{t \in[0, T]}\left|\mathcal{L}_{t}^{N}\left(x^{n}\right)\right|^{q}\right]<\infty
$$

for $q \geq 1$ and $n \in \mathbb{N}$ by using an induction argument on $n$.

To estimate the last term on the right-hand side of (3.17), we apply the Cauchy- 
Schwarz inequality to obtain, for $0 \leq k \leq n$,

$$
\begin{aligned}
& \mathbb{E}\left[\sup _{t \in[0, T]}\left|\frac{n+2}{2 N} \int_{0}^{t} \mathcal{L}_{s}^{N}\left(x^{n-k}\right) \mathcal{L}_{s}^{N}\left(x^{k+1}\right) d s\right|^{q}\right] \\
\leq & \frac{(n+2)^{q} T^{q}}{2^{q} N^{q}} \mathbb{E}\left[\sup _{t \in[0, T]}\left|\mathcal{L}_{t}^{N}\left(x^{n-k}\right)\right|^{q} \sup _{t \in[0, T]}\left|\mathcal{L}_{t}^{N}\left(x^{k+1}\right)\right|^{q}\right] \\
\leq & \frac{(n+2)^{q} T^{q}}{2^{q} N^{q}}\left\{\mathbb{E}\left[\sup _{t \in[0, T]}\left|\mathcal{L}_{t}^{N}\left(x^{n-k}\right)\right|^{2 q}\right] \mathbb{E}\left[\sup _{t \in[0, T]}\left|\mathcal{L}_{t}^{N}\left(x^{k+1}\right)\right|^{2 q}\right]\right\}^{1 / 2} \\
\leq & C(n, T, q) N^{-q},
\end{aligned}
$$

for some constant $C(n, T, q)$. Thus, the last term on the right-hand side of (3.17) converges to 0 in $L^{q}$ for $q>1$, as $N$ tends to infinity. By Markov inequality and Borel-Cantelli Lemma, one can also obtain the almost sure convergence.

If we define

$$
\begin{aligned}
\tilde{Q}_{t}^{N}\left(x^{n+2}\right)= & \mathcal{L}_{t}^{N}\left(x^{n+2}\right)-\mathcal{L}_{0}^{N}\left(x^{n+2}\right)-c(n+2) \int_{0}^{t} \mathcal{L}_{s}^{N}\left(x^{n+1}\right) d s \\
& -(n+2)(n+1) \int_{0}^{t}\left\langle x^{n+1}, \mu_{s}\right\rangle d s \\
& -(n+2) \sum_{k=0}^{n} \int_{0}^{t}\left(\mathcal{L}_{s}^{N}\left(x^{n-k}\right)\left\langle x^{k+1}, \mu_{s}\right\rangle+\mathcal{L}_{s}^{N}\left(x^{n+1-k}\right)\left\langle x^{k}, \mu_{s}\right\rangle\right) d s,
\end{aligned}
$$

for $n \geq-1$, then the difference $\left|\tilde{Q}_{t}^{N}\left(x^{n+2}\right)-Q_{t}^{N}\left(x^{n+2}\right)\right|$ converges to 0 almost surely and in $L^{q}$ for $q>1$. Thus, Corollary 2.3 implies that $\left(\tilde{Q}_{t}^{N}\left(x^{k}\right), \tilde{Q}_{t}^{N}\left(x^{k-1}\right), \ldots, \tilde{Q}_{t}^{N}(x)\right)_{t \in[0, T]}$ converges in distribution to $\left(G_{t}\left(x^{k}\right), G_{t}\left(x^{k-1}\right), \ldots, G_{t}(x)\right)_{t \in[0, T]}$ with covariance (3.16).

Now we deduce the convergence in distribution of $\left(\mathcal{L}_{t}^{N}\left(x^{k}\right)\right)_{t \in[0, T]}$ for $k \in \mathbb{N}$. First of all, we have $\mathcal{L}_{t}^{N}(1)=0$ and $\mathcal{L}_{t}^{N}(x)=\mathcal{L}_{0}^{N}(x)+\tilde{Q}_{t}^{N}(x)$ converges in distribution since the initial value converges in probability. By induction, if we assume $\left(\mathcal{L}_{t}^{N}\left(x^{k}\right), \ldots, \mathcal{L}_{t}^{N}(x)\right)_{t \in[0, T]}$ convergence in distribution to $\left(\mathcal{L}_{t}\left(x^{k}\right), \ldots, \mathcal{L}_{t}(x)\right)_{t \in[0, T]}$, then the convergence in distribution of $\left(\tilde{Q}_{t}^{N}\left(x^{k+1}\right), \tilde{Q}_{t}^{N}\left(x^{k}\right), \ldots, \tilde{Q}_{t}^{N}(x)\right)_{t \in[0, T]}$ implies the convergence in distribution of $\left(\tilde{Q}_{t}^{N}\left(x^{k+1}\right), \mathcal{L}_{t}^{N}\left(x^{k}\right), \ldots, \mathcal{L}_{t}^{N}(x)\right)_{t \in[0, T]}$, and hence $\left(\mathcal{L}_{t}^{N}\left(x^{k+1}\right), \ldots, \mathcal{L}_{t}^{N}(x)\right)_{t \in[0, T]}$ converges in distribution.

Thus, by (3.18) we have

$$
\begin{aligned}
G_{t}\left(x^{n+2}\right) \stackrel{d}{=} & \mathcal{L}_{t}\left(x^{n+2}\right)-\mathcal{L}_{0}\left(x^{n+2}\right) \\
& -c(n+2) \int_{0}^{t} \mathcal{L}_{s}\left(x^{n+1}\right) d s-(n+2)(n+1) \int_{0}^{t}\left\langle x^{n+1}, \mu_{s}\right\rangle d s \\
& -(n+2) \sum_{k=0}^{n} \int_{0}^{t}\left(\mathcal{L}_{s}\left(x^{n-k}\right)\left\langle x^{k+1}, \mu_{s}\right\rangle+\mathcal{L}_{s}\left(x^{n+1-k}\right)\left\langle x^{k}, \mu_{s}\right\rangle\right) d s,
\end{aligned}
$$

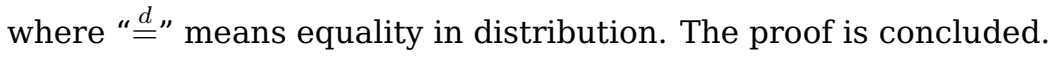

Remark 3.6. By the self-similarity of Brownian motion, when $X_{0}^{N}=0$, we have $X_{t}^{N} \stackrel{d}{=}$ $t X_{1}^{N}$. Thus, $\left(\lambda_{1}^{N}(t), \ldots, \lambda_{N}^{N}(t)\right) \stackrel{d}{=}\left(t \lambda_{1}^{N}(1), \ldots, t \lambda_{N}^{N}(1)\right)$. Therefore,

$$
\left\langle f(x), L_{N}(t)\right\rangle=\frac{1}{N} \sum_{i=1}^{N} f\left(\lambda_{i}^{N}(t)\right) \stackrel{d}{=} \frac{1}{N} \sum_{i=1}^{N} f\left(t \lambda_{i}^{N}(1)\right)=\left\langle f(t x), L_{N}(1)\right\rangle,
$$


and

$$
\left\langle f(x), \mu_{t}\right\rangle \stackrel{d}{=}\left\langle f(t x), \mu_{1}\right\rangle
$$

Hence, $\mathcal{L}_{t}^{N}(f(x)) \stackrel{d}{=} \mathcal{L}_{1}^{N}(f(t x))$, and thus, $\mathcal{L}_{t}(f(x)) \stackrel{d}{=} \mathcal{L}_{1}(f(t x))$. With these identities and the linearity of $\mathcal{L}_{t}(\cdot)$, (3.15) can be simplified as, for $n \geq 0$,

$$
\begin{aligned}
\mathcal{L}_{1}\left(x^{n+2}\right)= & c \mathcal{L}_{1}\left(x^{n+1}\right)+(n+1)\left\langle x^{n+1}, \mu_{1}\right\rangle \\
& +\sum_{k=0}^{n}\left(\mathcal{L}_{1}\left(x^{n-k}\right)\left\langle x^{k+1}, \mu_{1}\right\rangle+\mathcal{L}_{1}\left(x^{n+1-k}\right)\left\langle x^{k}, \mu_{1}\right\rangle\right)+\frac{1}{t^{n+2}} G_{t}\left(x^{n+2}\right), \quad t>0,
\end{aligned}
$$

where the Gaussian family $\left\{G_{t}\left(x^{n}\right), t \in[0, T]\right\}_{n \in \mathbb{N}}$ has the covariance functions

$$
\mathbb{E}\left[G_{t}\left(x^{n}\right) G_{s}\left(x^{m}\right)\right]=\frac{4 m n}{m+n}(t \wedge s)^{n+m}\left\langle x^{n+m-1}, \mu_{1}\right\rangle, \quad n, m \geq 1 .
$$

Note that the case $t=1$ corresponds to the classical Wishart matrix, and $\mu_{1}$ is the Marchenko-Pastur law. More precisely, recalling that $\mathcal{L}_{1}(1)=0$ and $\mathcal{L}_{1}(x)=G_{1}(x)$, we get by (3.19) $\mathcal{L}_{1}\left(x^{2}\right)=\left\langle x, \mu_{1}\right\rangle+(c+1) G_{1}(x)+G_{1}\left(x^{2}\right)$, for $m \geq 3$, and more generally $\mathcal{L}_{1}\left(x^{m}\right)=c_{m, 0}+c_{m, 1} G_{1}(x)+\ldots+c_{m, m} G_{1}\left(x^{m}\right)$ for some coefficients $\left(c_{m, j}\right)_{0 \leq j \leq m}$ which are determined recursively by (3.19).

We now study a more general particle system:

$$
d \lambda_{i}^{N}(t)=2 \frac{\sqrt{\lambda_{i}^{N}(t)}}{\sqrt{N}} d W_{i}(t)+\left(b_{N}\left(\lambda_{i}^{N}(t)\right)+\frac{1}{N} \sum_{j: j \neq i} \frac{\lambda_{i}^{N}(t)+\lambda_{j}^{N}(t)}{\lambda_{i}^{N}(t)-\lambda_{j}^{N}(t)}\right) d t, 1 \leq i \leq N, t \geq 0 .
$$

Compared to (3.5), the constant $P / N$ is replaced by a function $b_{N}(x)$ that will be assumed to converge to a constant $c$ in Corollary 3.7 below. Despite the extension being small, the system (3.20) may not have an explicit joint density function or/and stationary distribution, and hence cannot be treated in the same way as for the eigenvalues of Wishart process.

Corollary 3.7. Consider the SDEs (3.20), where $b_{N}(x)$ satisfies, for some constant $c \geq 1$,

$$
\lim _{N \rightarrow \infty} N\left\|b_{N}(x)-c\right\|_{L^{\infty}(\mathbb{R})}=0 .
$$

Assume the same initial conditions as in Theorem 3.5. Then the conclusion of Theorem 3.5 still holds.

Proof. Let $p_{1}=N\left(c-\left\|b_{N}(x)-c\right\|_{L^{\infty}}\right)$ and $p_{2}=N\left(c+\left\|b_{N}(x)-c\right\|_{L^{\infty}}\right)$ be two constants depending on $N$. Then (3.21) implies $p_{2} \geq p_{1}>N-1$ when $N$ is large. Clearly, $p_{1} \leq N\left\|b_{N}(x)\right\|_{L^{\infty}(\mathbb{R})} \leq p_{2}$. Consider the following two systems of SDEs:

$$
d x_{i}^{N}(t)=2 \frac{\sqrt{x_{i}^{N}(t)}}{\sqrt{N}} d W_{i}(t)+\left(\frac{p_{1}}{N}+\frac{1}{N} \sum_{j: j \neq i} \frac{x_{i}^{N}(t)+x_{j}^{N}(t)}{x_{i}^{N}(t)-x_{j}^{N}(t)}\right) d t, 1 \leq i \leq N, t \geq 0,
$$

and

$$
d y_{i}^{N}(t)=2 \frac{\sqrt{y_{i}^{N}(t)}}{\sqrt{N}} d W_{i}(t)+\left(\frac{p_{2}}{N}+\frac{1}{N} \sum_{j: j \neq i} \frac{y_{i}^{N}(t)+y_{j}^{N}(t)}{y_{i}^{N}(t)-y_{j}^{N}(t)}\right) d t, 1 \leq i \leq N, t \geq 0,
$$


with the initial conditions $x_{i}^{N}(0)=y_{i}^{N}(0)=\lambda_{i}^{N}(0)$. By the comparison principle in Corollary 3.2, we have

$$
\mathbb{P}\left(x_{i}^{N}(t) \leq \lambda_{i}^{N}(t) \leq y_{i}^{N}(t), \forall t \geq 0, \forall 1 \leq i \leq N\right)=1 .
$$

Thus, almost surely,

$$
\begin{aligned}
\sup _{t \in[0, T]}\left\langle|x|^{p}, L_{N}(t)\right\rangle & =\sup _{t \in[0, T]} \frac{1}{N} \sum_{i=1}^{N}\left|\lambda_{i}^{N}(t)\right|^{p} \\
& \leq \sup _{t \in[0, T]} \frac{1}{N} \sum_{i=1}^{N}\left(\left|x_{i}^{N}(t)\right|^{p}+\left|y_{i}^{N}(t)\right|^{p}\right) \\
& \leq \sup _{t \in[0, T]}\left\langle|x|^{p}, L_{N}^{(x)}(t)\right\rangle+\sup _{t \in[0, T]}\left\langle|x|^{p}, L_{N}^{(y)}(t)\right\rangle,
\end{aligned}
$$

where $\left\{L_{N}^{(x)}(t), t \in[0, T]\right\}_{N \in \mathbb{N}}$ and $\left\{L_{N}^{(y)}(t), t \in[0, T]\right\}_{N \in \mathbb{N}}$ are the empirical measures of the two particle systems $\left(x_{i}^{N}(t)\right)_{1 \leq i \leq N}$ and $\left(y_{i}^{N}(t)\right)_{1 \leq i \leq N}$, respectively.

Noting that $p_{1} / N$ and $p_{2} / N$ converge to $c$ as $N \rightarrow \infty$ by (3.21), we have that Lemma 3.4 holds for the two systems (3.22) and (3.23), and thus also holds for (3.20) by (3.24). Furthermore, condition (3.21) also yields that $b_{N}(x) \rightarrow c$ uniformly as $N \rightarrow \infty$, and hence (3.17) still holds. Then the rest of the proof follows that of Theorem 3.5.

\subsection{Application to Dyson's Brownian motion}

In this subsection, we discuss the CLT for Dyson's Brownian motion. It was shown in Anderson et al. (2010); Graczyk and Małecki (2014); Song et al. (2020), the scaled symmetric matrix-valued Brownian motion $X_{t}^{N}=\left(\tilde{B}^{\top}(t)+\tilde{B}(t)\right) / \sqrt{2 N}$, where $\tilde{B}(t)$ is a $N \times N$ matrix-valued Brownian motion, is the solution of the matrix SDE (1.1) with the coefficient functions

$$
g_{N}(x) h_{N}(y)=\frac{1}{\sqrt{2 N}}, \quad b_{N}(x)=0 .
$$

The system of SDEs of the eigenvalue processes, that is, Dyson's Brownian motion, is

$$
d \lambda_{i}^{N}(t)=\frac{\sqrt{2}}{\sqrt{N}} d W_{i}(t)+\frac{1}{N} \sum_{j: j \neq i} \frac{1}{\lambda_{i}^{N}(t)-\lambda_{j}^{N}(t)} d t, 1 \leq i \leq N, t \geq 0 .
$$

Hence, the eigenvalue processes are the particles in (1.6) with

$$
\sigma^{N}(x)=\frac{\sqrt{2}}{\sqrt{N}}, b_{N}(x)=0, H_{N}(x, y)=\frac{1}{N},
$$

and thus,

$$
N H_{N}(x, y)=H(x, y)=1, b(x)=0, \sqrt{N} \sigma^{N}(x)=\tilde{\sigma}(x)=\sqrt{2} .
$$

Here, we consider the distribution $\mathbb{Q}^{N}$ on $\Delta_{N}^{\prime}=\left\{x=\left(x_{1}, x_{2}, \ldots, x_{N}\right) \in \mathbb{R}^{N}: x_{1}<\right.$ $\left.\ldots<x_{N}\right\}$ with the density function

$$
C_{N} \exp \left(-\frac{N}{4} \sum_{i=1}^{N} x_{i}^{2}\right) \prod_{1 \leq j<i \leq N}\left|x_{i}-x_{j}\right|,
$$

where $C_{N}$ is a normalization constant.

Similar to Wishart process, we can obtain the following central limit theorem. 
Theorem 3.8. Let $\xi^{N}=\left(\xi_{1}^{N}, \ldots, \xi_{N}^{N}\right)$ be a random vector independent of $\left(W_{1}, \ldots, W_{N}\right)$ with (3.27) as its joint probability density function. Assume that $\left(\lambda_{1}^{N}(0), \ldots, \lambda_{N}^{N}(0)\right)$ is independent of $\left(W_{1}, \ldots, W_{N}\right)$ and that there exist constants $a, b \geq 0$, such that

$$
\sqrt{a} \xi_{i}^{N}-b \leq \lambda_{i}^{N}(0) \leq \sqrt{a} \xi_{i}^{N}+b
$$

for $1 \leq i \leq N$ almost surely. Besides, assume that for any polynomial $f(x) \in \mathbb{R}[x]$, the initial value $\mathcal{L}_{0}^{N}(f)$ converges in probability to a random variable $\mathcal{L}_{0}(f)$. Furthermore, assume that for all $n \in \mathbb{N}$,

$$
\sup _{N} \mathbb{E}\left[\left|N\left(\left\langle x^{n}, L_{N}(0)\right\rangle-\left\langle x^{n}, \mu_{0}\right\rangle\right)\right|^{p}\right]<\infty
$$

for all $p \geq 1$.

Then for any $0<T<\infty$, there exists a family of processes $\left\{\mathcal{L}_{t}(f), t \in[0, T]\right\}_{f \in \mathbb{R}[x]}$, such that for any $n \in \mathbb{N}$ and any polynomials $P_{1}, \ldots, P_{n} \in \mathbb{R}[x]$, the vector-valued process $\left(\mathcal{L}_{t}^{N}\left(P_{1}\right), \ldots, \mathcal{L}_{t}^{N}\left(P_{n}\right)\right)_{t \in[0, T]}$ converges to $\left(\mathcal{L}_{t}\left(P_{1}\right), \ldots, \mathcal{L}_{t}\left(P_{n}\right)\right)_{t \in[0, T]}$ in distribution.

The limit processes $\left\{\mathcal{L}_{t}(f), t \in[0, T]\right\}_{f \in \mathbb{R}[x]}$ are characterized by the following properties.

1. For $P_{1}, P_{2} \in \mathbb{R}[x], \alpha_{1}, \alpha_{2} \in \mathbb{R}, t \in[0, T]$,

$$
\mathcal{L}_{t}\left(\alpha_{1} P_{1}+\alpha_{2} P_{2}\right)=\alpha_{1} \mathcal{L}_{t}\left(P_{1}\right)+\alpha_{2} \mathcal{L}_{t}\left(P_{2}\right) .
$$

2. The basis $\left\{\mathcal{L}_{t}\left(x^{n}\right), t \in[0, T]\right\}_{n \in \mathbb{N}}$ of $\left\{\mathcal{L}_{t}(f), t \in[0, T]\right\}_{f \in \mathbb{R}[x]}$ satisfies

$$
\mathcal{L}_{t}(1)=0, \quad \mathcal{L}_{t}(x)=\mathcal{L}_{0}(x)+G_{t}(x),
$$

and for $n \geq 0$,

$$
\begin{aligned}
\mathcal{L}_{t}\left(x^{n+2}\right)= & \mathcal{L}_{0}\left(x^{n+2}\right)+\frac{(n+2)(n+1)}{2} \int_{0}^{t}\left\langle x^{n}, \mu_{s}\right\rangle d s \\
& +(n+2) \sum_{k=0}^{n} \int_{0}^{t} \mathcal{L}_{s}\left(x^{n-k}\right)\left\langle x^{k}, \mu_{s}\right\rangle d s+G_{t}\left(x^{n+2}\right),
\end{aligned}
$$

where $\left\{G_{t}\left(x^{n}\right), t \in[0, T]\right\}_{n \in \mathbb{N}}$ is a centered Gaussian family with the covariance

$$
\mathbb{E}\left[G_{t}\left(x^{n}\right) G_{s}\left(x^{m}\right)\right]=2 m n \int_{0}^{t \wedge s}\left\langle x^{n+m-2}, \mu_{u}\right\rangle d u, \quad n, m \geq 1 .
$$

Proof. The proof is similar to the proofs of the Wishart case (Lemma 3.3, Lemma 3.4 and Theorem 3.5), which is sketched below.

Consider the following SDE, for $1 \leq i \leq N$,

$$
d u_{i}^{N}(t)=\frac{\sqrt{2}}{\sqrt{N(t+a)}} d W_{i}(t)+\frac{1}{t+a}\left(-\frac{1}{2} u_{i}^{N}(t)+\frac{1}{N} \sum_{j: j \neq i} \frac{1}{u_{i}^{N}(t)-u_{j}^{N}(t)}\right) d t, \quad t \geq 0 .
$$

Then $\frac{d}{d t} \mathbb{E}\left[f\left(u^{N}(t)\right)\right]$ vanishes for any $f \in C_{b}^{2}(\mathbb{R})$ if $u^{N}(t)$ has the distribution $\mathbb{Q}^{N}$ given in (3.27), and hence the process $u^{N}(t)$ with initial value $u^{N}(0)=\xi^{N}$ is stationary (see (Anderson et al., 2010, Lemma 4.3.17)). Let $v_{i}^{N}(t)=\sqrt{t+a} u_{i}^{N}(t)+b$ for $1 \leq i \leq N$. Then $v^{N}(t)$ and $\lambda^{N}(t)$ solve the same SDEs (3.25), and by the comparison principle in Corollary 3.2, we have

$$
\lambda_{i}^{N}(t) \leq v_{i}^{N}(t)=\sqrt{t+a} u_{i}^{N}(t)+b
$$


A similar argument leads to

$$
-\lambda_{i}^{N}(t) \leq-\sqrt{t+a} u_{i}^{N}(t)+b
$$

Therefore,

$$
\left|\lambda_{i}^{N}(t)\right| \leq \sqrt{t+a}\left|u_{i}^{N}(t)\right|+b
$$

Using the tail probability estimation based on the density function (3.27) of $u_{i}^{N}(t)$,

$$
\mathbb{P}\left(\left|u_{i}^{N}(t)\right| \geq x\right) \leq \mathbb{P}\left(u_{N}^{N}(t) \geq x\right)+\mathbb{P}\left(u_{1}^{N}(t) \leq-x\right) \leq 2 \mathbb{P}\left(u_{N}^{N}(t) \geq x\right) \leq \exp (-\alpha N x),
$$

where $\alpha$ is positive constant independent of $N$, we obtain

$$
\begin{aligned}
\mathbb{E}\left[\left|\lambda_{i}^{N}(t)\right|^{k}\right] & \leq \mathbb{E}\left[\left(\sqrt{t+a}\left|u_{i}^{N}(t)\right|+b\right)^{k}\right] \\
& \leq 2^{k} \sqrt{t+a}^{k} \mathbb{E}\left[\left|u_{i}^{N}(t)\right|^{k}\right]+2^{k} b^{k} \\
& =2^{k} \sqrt{t+a}^{k} k \int_{0}^{\infty} x^{k-1} \mathbb{P}\left(\left|u_{i}^{N}(t)\right| \geq x\right) d x+2^{k} b^{k} \\
& \leq 2^{k} \sqrt{t+a}^{k} k \int_{0}^{\infty} x^{k-1} \exp (-\alpha N x) d x+2^{k} b^{k} \\
& =2^{k} \sqrt{t+a}^{k} \frac{\Gamma(k+1)}{(\alpha N)^{k}}+2^{k} b^{k} \\
& \leq 2^{k} \sqrt{t+a}^{k}+2^{k} b^{k} \\
& \leq 2\left(4 b^{2}+4(t+a)\right)^{k / 2}
\end{aligned}
$$

for $k \in[0, \alpha N]$. Then a similar argument in the proof of Lemma 3.4 leads to

$$
\mathbb{E}\left[\sup _{t \in[0, T]}\left\langle|x|^{p}, L_{N}(t)\right\rangle\right] \leq C(a, b, T)^{p}
$$

for some positive constant $C(a, b, T)$ depending only on $(a, b, T)$ and all $p \geq 0, N \geq \alpha p$ for some positive constant $\alpha$.

Then applying Corollary 2.5 and following the approach in the proof of Theorem 3.5, we may get the desired result.

Remark 3.9. A CLT was obtained in (Anderson et al., 2010, Theorem 4.3.20) for Dyson's Brownian motion with bounded initial values. (We would like to point out that there should be a constant factor $2 / \beta$ in the covariance function which equals to 2 in the real case and equals to 1 in the complex case in Anderson et al. (2010).) Thanks to the comparison principle Corollary 3.2, the CLT also applies to Dyson's Brownian motion with possibly unbounded initial values satisfying (3.28), as stated in Theorem 3.8. For example, the CLT is valid for Dyson's Brownian motion with unbounded initial value $\xi^{N}$. Note that $\xi^{N}$ has the joint density (3.27), which is also the joint density of $\left\{\lambda_{i}(1)\right\}_{1 \leq i \leq N}$, assuming $\left\{\lambda_{i}(t)\right\}_{1 \leq i \leq N}$ is a Dyson's Brownian motion starting at 0 .

Similar to the Wishart case, the self-similarity of the Brownian motion implies $\mathcal{L}_{t}(f(x)) \stackrel{d}{=} \mathcal{L}_{1}(f(\sqrt{t} x))$ and $\left\langle f(x), \mu_{t}\right\rangle=\left\langle f(\sqrt{t} x), \mu_{1}\right\rangle$ when the initial value $X_{0}^{N}=0$. Thus, (3.29) can be simplified as, for $n \geq 0$,

$$
\mathcal{L}_{1}\left(x^{n+2}\right)=(n+1)\left\langle x^{n}, \mu_{1}\right\rangle+2 \sum_{k=0}^{n} \mathcal{L}_{1}\left(x^{n-k}\right) \mu_{1}\left(x^{k}\right)+\frac{1}{t^{\frac{n+2}{2}}} G_{t}\left(x^{n+2}\right), \quad t>0,
$$


with covariance functions

$$
\mathbb{E}\left[G_{t}\left(x^{n}\right) G_{s}\left(x^{m}\right)\right]=\frac{4 m n}{m+n}(t \wedge s)^{\frac{m+n}{2}}\left\langle x^{m+n-2}, \mu_{1}\right\rangle, \quad n, m \geq 1 .
$$

The case $t=1$ corresponds to the classical GOE matrix, and $\mu_{1}$ is the semicircle law. Some beginning terms are $\mathcal{L}_{1}(1)=0, \mathcal{L}_{1}(x)=G_{1}(x)$ and $\mathcal{L}_{1}\left(x^{2}\right)=1+G_{1}\left(x^{2}\right)$. By (3.31), for $m \geq 2, \mathcal{L}_{1}\left(x^{m}\right)$ has the distribution of a linear combination of central Gaussian variables $\left\{G_{1}\left(x^{j}\right), 1 \leq j \leq m\right\}$.

The following Corollary extends the result of Theorem 3.8 by allowing asymptotical constant drift coefficient functions.

Corollary 3.10. Consider the following SDES

$$
d \lambda_{i}^{N}(t)=\frac{\sqrt{2}}{\sqrt{N}} d W_{i}(t)+\left(b_{N}\left(\lambda_{i}^{N}(t)\right)+\frac{1}{N} \sum_{j: j \neq i} \frac{1}{\lambda_{i}^{N}(t)-\lambda_{j}^{N}(t)}\right) d t, 1 \leq i \leq N, t \geq 0,
$$

where $b_{N}(x)$ satisfies, for some constant $c \in \mathbb{R}$,

$$
\lim _{N \rightarrow \infty} N\left\|b_{N}(x)-c\right\|_{L^{\infty}(\mathbb{R})}=0
$$

Furthermore, assume the same initial conditions as in Theorem 3.8. Then the conclusion of Theorem 3.8 still holds with (3.29) replaced by

$$
\begin{aligned}
\mathcal{L}_{t}\left(x^{n+2}\right)= & \mathcal{L}_{0}\left(x^{n+2}\right)+c(n+2) \int_{0}^{t} \mathcal{L}_{s}\left(x^{n+1}\right) d s+\frac{(n+2)(n+1)}{2} \int_{0}^{t}\left\langle x^{n}, \mu_{s}\right\rangle d s \\
& +(n+2) \sum_{k=0}^{n} \int_{0}^{t} \mathcal{L}_{s}\left(x^{n-k}\right) \mu_{s}\left(x^{k}\right) d s+G_{t}\left(x^{n+2}\right),
\end{aligned}
$$

for $n \geq-1$.

Proof. Set $c_{1}=c-1$ and $c_{2}=c+1$. Then by (3.33), there exists $N_{0} \in \mathbb{N}$ such that for $N \geq N_{0}, c_{1} \leq\left\|b_{N}(x)\right\|_{L^{\infty}(\mathbb{R})} \leq c_{2}$. Without loss of generality, we assume $c_{1} \leq$ $\left\|b_{N}(x)\right\|_{L^{\infty}(\mathbb{R})} \leq c_{2}$ for all $N \geq 1$.

Consider the following two systems of SDEs:

$$
d x_{i}^{N}(t)=\frac{\sqrt{2}}{\sqrt{N}} d W_{i}(t)+\left(c_{1}+\frac{1}{N} \sum_{j: j \neq i} \frac{1}{x_{i}^{N}(t)-x_{j}^{N}(t)}\right) d t, 1 \leq i \leq N, t \geq 0
$$

and

$$
d y_{i}^{N}(t)=\frac{\sqrt{2}}{\sqrt{N}} d W_{i}(t)+\left(c_{2}+\frac{1}{N} \sum_{j: j \neq i} \frac{1}{y_{i}^{N}(t)-y_{j}^{N}(t)}\right) d t, 1 \leq i \leq N, t \geq 0
$$

with the initial conditions $x_{i}^{N}(0)=y_{i}^{N}(0)=\lambda_{i}^{N}(0)$ for $1 \leq i \leq N$. By the comparison principle Theorem 3.1, we have

$$
\mathbb{P}\left(x_{i}^{N}(t) \leq \lambda_{i}^{N}(t) \leq y_{i}^{N}(t), \forall 1 \leq i \leq N, \forall t>0\right)=1
$$


Thus, for $p \geq 1$, we have

$$
\begin{aligned}
& \sup _{t \in[0, T]}\left\langle|x|^{p}, L_{N}(t)\right\rangle=\sup _{t \in[0, T]} \frac{1}{N} \sum_{i=1}^{N}\left|\lambda_{i}^{N}(t)\right|^{p} \leq \sup _{t \in[0, T]} \frac{1}{N} \sum_{i=1}^{N}\left(\left|x_{i}^{N}(t)\right|^{p}+\left|y_{i}^{N}(t)\right|^{p}\right) \\
& \leq \sup _{t \in[0, T]} \frac{1}{N} \sum_{i=1}^{N} 2^{p}\left(\left|x_{i}^{N}(t)-c_{1} t\right|^{p}+\left(c_{1} t\right)^{p}+\left|y_{i}^{N}(t)-c_{2} t\right|^{p}+\left(c_{2} t\right)^{p}\right) \\
& \leq 2^{p}\left(\sup _{t \in[0, T]}\left\langle|x|^{p}, L_{N}^{(x)}(t)\right\rangle+\sup _{t \in[0, T]}\left\langle|x|^{p}, L_{N}^{(y)}(t)\right\rangle+\left(c_{1} T\right)^{p}+\left(c_{2} T\right)^{p}\right),
\end{aligned}
$$

almost surely, where $\left\{L_{N}^{(x)}(t), t \in[0, T]\right\}_{N \in \mathbb{N}}$ and $\left\{L_{N}^{(y)}(t), t \in[0, T]\right\}_{N \in \mathbb{N}}$ are the empirical measures of the two particle systems $\left(x_{i}^{N}(t)-c_{1} t\right)_{1 \leq i \leq N}$ and $\left(y_{i}^{N}(t)-c_{2} t\right)_{1 \leq i \leq N}$, respectively.

It is easy to verify that both $\left(x_{i}^{N}(t)-c_{1} t\right)_{1 \leq i \leq N}$ and $\left(y_{i}^{N}(t)-c_{2} t\right)_{1 \leq i \leq N}$ solve Dyson's SDEs (3.25). By (3.30) in the proof Theorem 3.8, we have

$$
\mathbb{E}\left[\sup _{t \in[0, T]}\left\langle|x|^{p}, L_{N}^{(x)}(t)\right\rangle\right] \leq C(a, b, T)^{p} \text { and } \mathbb{E}\left[\sup _{t \in[0, T]}\left\langle|x|^{p}, L_{N}^{(y)}(t)\right\rangle\right] \leq C(a, b, T)^{p},
$$

and consequently, by (3.37)

$$
\mathbb{E}\left[\sup _{t \in[0, T]}\left\langle|x|^{p}, L_{N}(t)\right\rangle\right] \leq C(a, b, T)^{p}
$$

for some positive constant $C(a, b, T)$ depending only on $(a, b, T)$ and all $p \geq 1, N \geq \alpha p$ for some positive constant $\alpha$.

Note that (3.33) also implies that $b_{N}(x)$ converges to the constant $c$ uniformly as $N \rightarrow \infty$. Then applying Corollary 2.5 and following the approach in the proof of Theorem 3.5, we get the desired result.

\subsection{Application to eigenvalues of symmetric matrix-valued OU matrix}

In this subsection, we discuss the CLT for the eigenvalues of a symmetric matrixvalued Ornstein-Uhlenbeck process. It was shown in Chan (1992), the symmetric $N \times N$ matrix $X^{N}(t)$, whose entries $\left\{X_{i j}^{N}(t), i \leq j\right\}$ are independent Ornstein-Uhlenbeck processes with invariant distribution $N\left(0,\left(1+\delta_{i j}\right) /(2 N)\right)$, where $\delta_{i j}$ is the Kronecker delta function, is the solution of the matrix $\operatorname{SDE}$ (1.1) with the coefficient functions

$$
g_{N}(x) h_{N}(y)=\frac{1}{2 \sqrt{N}}, \quad b_{N}(x)=-\frac{1}{2} x .
$$

The SDEs of the eigenvalue processes are

$$
d \lambda_{i}^{N}(t)=\frac{1}{\sqrt{N}} d W_{i}(t)+\left(-\frac{1}{2} \lambda_{i}^{N}(t)+\frac{1}{2 N} \sum_{j: j \neq i} \frac{1}{\lambda_{i}^{N}(t)-\lambda_{j}^{N}(t)}\right) d t, 1 \leq i \leq N, t \geq 0 .
$$

Hence, the eigenvalue processes are the particles in (1.6) with

$$
\sigma^{N}(x)=\frac{1}{\sqrt{N}}, b_{N}(x)=-\frac{1}{2} x, H_{N}(x, y)=\frac{1}{2 N},
$$

and thus,

$$
N H_{N}(x, y)=H(x, y)=\frac{1}{2}, b(x)=-\frac{1}{2} x, \sqrt{N} \sigma^{N}(x)=\tilde{\sigma}(x)=1 .
$$


Similar to the eigenvalues of Wishart process and Dyson's Brownian motion, we have the following CLT.

Theorem 3.11. Let $\xi^{N}=\left(\xi_{1}^{N}, \ldots, \xi_{N}^{N}\right)$ be a random vector that is independent of $\left(W_{1}, \ldots, W_{N}\right)$ and has (3.27) as its joint probability density function. Assume that $\left(\lambda_{1}^{N}(0), \ldots, \lambda_{N}^{N}(0)\right)$ is independent of $\left(W_{1}, \ldots, W_{N}\right)$ and that there exist constants $a, b \geq 0$, such that

$$
\sqrt{a} \xi_{i}^{N}-b \leq \lambda_{i}^{N}(0) \leq \sqrt{a} \xi_{i}^{N}+b
$$

for $1 \leq i \leq N$ almost surely.

Besides, assume that for any polynomial $f(x) \in \mathbb{R}[x]$, the initial value $\mathcal{L}_{0}^{N}(f)$ converges in probability to a random variable $\mathcal{L}_{0}(f)$. Furthermore, assume that for all $n \in \mathbb{N}$,

$$
\sup _{N} \mathbb{E}\left[\left|N\left(\left\langle x^{n}, L_{N}(0)\right\rangle-\left\langle x^{n}, \mu_{0}\right\rangle\right)\right|^{p}\right]<\infty
$$

for all $p \geq 1$.

Then for any $0<T<\infty$, there exists a family of processes $\left\{\mathcal{L}_{t}(f), t \in[0, T]\right\}_{f \in \mathbb{R}[x]}$, such that for any $n \in \mathbb{N}$ and any polynomials $P_{1}, \ldots, P_{n} \in \mathbb{R}[x]$, the vector-valued process $\left(\mathcal{L}_{t}^{N}\left(P_{1}\right), \ldots, \mathcal{L}_{t}^{N}\left(P_{n}\right)\right)_{t \in[0, T]}$ converges to $\left(\mathcal{L}_{t}\left(P_{1}\right), \ldots, \mathcal{L}_{t}\left(P_{n}\right)\right)_{t \in[0, T]}$ in distribution.

The limit processes $\left\{\mathcal{L}_{t}(f), t \in[0, T]\right\}_{f \in \mathbb{R}[x]}$ are characterized by the following properties.

1. For $P_{1}, P_{2} \in \mathbb{R}[x], \alpha_{1}, \alpha_{2} \in \mathbb{R}, t \in[0, T]$,

$$
\mathcal{L}_{t}\left(\alpha_{1} P_{1}+\alpha_{2} P_{2}\right)=\alpha_{1} \mathcal{L}_{t}\left(P_{1}\right)+\alpha_{2} \mathcal{L}_{t}\left(P_{2}\right)
$$

2. The basis $\left\{\mathcal{L}_{t}\left(x^{n}\right), t \in[0, T]\right\}_{n \in \mathbb{N}}$ of $\left\{\mathcal{L}_{t}(f), t \in[0, T]\right\}_{f \in \mathbb{R}[x]}$ satisfies

$$
\mathcal{L}_{t}(1)=0, \quad \mathcal{L}_{t}(x)=-\mathcal{L}_{0}(x)+G_{t}(x)-\frac{1}{2} e^{-t / 2} \int_{0}^{t} e^{s / 2}\left(G_{s}(x)-\mathcal{L}_{0}(x)\right) d s
$$

and for $n \geq 0$,

$$
\begin{aligned}
\mathcal{L}_{t}\left(x^{n+2}\right)= & e^{-\frac{n+2}{2} t} \mathcal{L}_{0}\left(x^{n+2}\right)+R_{t}(n)+G_{t}\left(x^{n+2}\right) \\
& -\frac{n+2}{2} e^{-\frac{n+2}{2} t} \int_{0}^{t} e^{\frac{n+2}{2} s}\left(R_{s}(n)+G_{s}\left(x^{n+2}\right)\right) d s .
\end{aligned}
$$

where

$$
R_{t}(n)=\frac{(n+2)(n+1)}{4} \int_{0}^{t}\left\langle x^{n}, \mu_{s}\right\rangle d s+\frac{n+2}{2} \sum_{k=0}^{n} \int_{0}^{t} \mathcal{L}_{s}\left(x^{n-k}\right)\left\langle x^{k}, \mu_{s}\right\rangle d s
$$

and $\left\{G_{t}\left(x^{n}\right), t \in[0, T]\right\}_{n \in \mathbb{N}}$ is a centered Gaussian family with the covariance

$$
\mathbb{E}\left[G_{t}\left(x^{n}\right) G_{s}\left(x^{m}\right)\right]=m n \int_{0}^{t \wedge s}\left\langle x^{n+m-2}, \mu_{u}\right\rangle d u, \quad n, m \geq 1 .
$$

Proof. Consider the symmetric matrix-valued OU matrix $X_{t}^{N}$, of which the entries $\left\{X_{i j}^{N}(t)\right\}$ satisfy

$$
d X_{i j}^{N}(t)=-\frac{1}{2} X_{i j}^{N}(t) d t+\frac{2 \delta_{i j}+\sqrt{2}\left(1-\delta_{i j}\right)}{2 \sqrt{N}} d B_{i j}(t), \quad 1 \leq i \leq j \leq N, t \geq 0
$$


where $\left\{B_{i j}(t), i \leq j\right\}$ is a family of independent Brownian motions. Denoting by

$$
\sigma_{i j}=\frac{2 \delta_{i j}+\sqrt{2}\left(1-\delta_{i j}\right)}{2 \sqrt{N}}
$$

the solution to (3.42) is given by

$$
X_{i j}^{N}(t)=X_{i j}^{N}(0) e^{-t / 2}+\sigma_{i j} e^{-t / 2} \int_{0}^{t} e^{s / 2} d B_{i j}(s) .
$$

The stochastic integral is a martingale with quadratic variation

$$
\left\langle\int_{0} e^{s / 2} d B_{i j}(s)\right\rangle_{t}=e^{t}-1
$$

By Knight's Theorem, there exists a family of independent standard one-dimensional Brownian motions $\left\{\tilde{B}_{i j}(t), i \leq j\right\}$, such that

$$
\int_{0}^{t} e^{s / 2} d B_{i j}(s)=\tilde{B}_{i j}\left(e^{t}-1\right) .
$$

Thus, we have

$$
X_{i j}^{N}(t)=e^{-t / 2}\left(X_{i j}^{N}(0)+\sigma_{i j} \tilde{B}_{i j}\left(e^{t}-1\right)\right) .
$$

Let $Y_{t}^{N}$ be a symmetric matrix-valued stochastic process whose entries $\left\{Y_{i j}^{N}(t), i \leq j\right\}$ are given by

$$
Y_{i j}^{N}(t)=Y_{i j}^{N}(0)+\sqrt{2} \sigma_{i j} \tilde{B}_{i j}(t),
$$

with $Y_{i j}^{N}(0)=\sqrt{2} X_{i j}^{N}(0), 1 \leq i \leq j \leq N$. Then $Y^{N}$ is the scaled symmetric matrix-valued Brownian motion introduced in section 3.3. By (3.43) and (3.44),

$$
\sqrt{2} e^{t / 2} X_{i j}^{N}(t)=Y_{i j}^{N}\left(e^{t}-1\right), 1 \leq i \leq j \leq N,
$$

and hence

$$
\sqrt{2} e^{t / 2} \lambda_{i}^{N}(t)=\tilde{\lambda}_{i}^{N}\left(e^{t}-1\right), 1 \leq i \leq N,
$$

where $\left\{\lambda_{i}^{N}(t)\right\}_{1 \leq i \leq N}$ and $\left\{\tilde{\lambda}_{i}^{N}(t)\right\}_{1 \leq i \leq N}$ are the eigenvalues of $X^{N}(t)$ and $Y^{N}(t)$, respectively.

Thus, almost surely, we have

$$
\begin{aligned}
\left\langle|x|^{p}, L_{N}(t)\right\rangle & =\frac{1}{N} \sum_{i=1}^{N}\left|\lambda_{i}^{N}(t)\right|^{p} \\
& =2^{-p / 2} e^{-p t / 2} \frac{1}{N} \sum_{i=1}^{N}\left|\tilde{\lambda}_{i}^{N}\left(e^{t}-1\right)\right|^{p} \\
& =2^{-p / 2} e^{-p t / 2}\left\langle|x|^{p}, \tilde{L}_{N}\left(e^{t}-1\right)\right\rangle, \forall t>0,
\end{aligned}
$$

where $L_{N}(t)$ and $\tilde{L}_{N}(t)$ are the empirical measures of $\left\{\lambda_{i}^{N}(t)\right\}_{1 \leq i \leq N}$ and $\left\{\tilde{\lambda}_{i}^{N}(t)\right\}_{1 \leq i \leq N}$, respectively. Note that $\tilde{\lambda}_{i}^{N}(0)=\sqrt{2} \lambda_{i}^{N}(0)$ satisfies condition (3.28) in Theorem 3.8 with the constants $a$ and $b$ replaced by $2 a$ and $\sqrt{2} b$. By the estimation (3.30), for all $p \geq 1$ and $N \geq \alpha p$ for some positive constant $\alpha$, we have

$$
\begin{aligned}
\mathbb{E}\left[\sup _{t \in[0, T]}\left\langle|x|^{p}, L_{N}(t)\right\rangle\right] & \leq 2^{-p / 2} \mathbb{E}\left[\sup _{t \in\left[0, e^{T}-1\right]}\left\langle|x|^{p}, \tilde{L}_{N}(t)\right\rangle\right] \\
& \leq 2^{-p / 2} C\left(2 a, \sqrt{2} b, e^{T}-1\right)^{p} \\
& =C^{\prime}(a, b, T)^{p},
\end{aligned}
$$


where $C^{\prime}(a, b, T)$ is positive constant depending only on $(a, b, T)$.

Thus, by Lemma 3.4 and Corollary 2.5, $Q_{t}^{N}\left(x^{n}\right)$ defined by (1.10) converges in distribution to a centered Gaussian family $\left\{G_{t}\left(x^{n}\right), t \in[0, T]\right\}_{n \in \mathbb{N}}$ with covariance given by (3.41). Similar to (3.17), for $n \geq-1$, we have

$$
\begin{aligned}
Q_{t}^{N}\left(x^{n+2}\right)= & \mathcal{L}_{t}^{N}\left(x^{n+2}\right)-\mathcal{L}_{0}^{N}\left(x^{n+2}\right)+\frac{n+2}{2} \int_{0}^{t} \mathcal{L}_{s}^{N}\left(x^{n+2}\right) d s-\frac{(n+2)(n+1)}{4} \int_{0}^{t}\left\langle x^{n}, \mu_{s}\right\rangle d s \\
& -\frac{n+2}{2} \sum_{k=0}^{n} \int_{0}^{t} \mathcal{L}_{s}^{N}\left(x^{n-k}\right)\left\langle x^{k}, \mu_{s}\right\rangle d s-\frac{(n+2)}{4 N} \sum_{k=0}^{n} \int_{0}^{t} \mathcal{L}_{s}^{N}\left(x^{n-k}\right) \mathcal{L}_{s}^{N}\left(x^{k}\right) d s .
\end{aligned}
$$

Letting $N \rightarrow \infty$, we have

$$
\begin{aligned}
G_{t}\left(x^{n+2}\right) \stackrel{d}{=} & \mathcal{L}_{t}\left(x^{n+2}\right)-\mathcal{L}_{0}\left(x^{n+2}\right)+\frac{n+2}{2} \int_{0}^{t} \mathcal{L}_{s}\left(x^{n+2}\right) d s-\frac{(n+2)(n+1)}{4} \int_{0}^{t}\left\langle x^{n}, \mu_{s}\right\rangle d s \\
& -\frac{n+2}{2} \sum_{k=0}^{n} \int_{0}^{t} \mathcal{L}_{s}\left(x^{n-k}\right)\left\langle x^{k}, \mu_{s}\right\rangle d s \\
= & \mathcal{L}_{t}\left(x^{n+2}\right)-\mathcal{L}_{0}\left(x^{n+2}\right)+\frac{n+2}{2} \int_{0}^{t} \mathcal{L}_{s}\left(x^{n+2}\right) d s-R_{t}(n)
\end{aligned}
$$

where $R_{t}(n)$ is given in (3.40). Without loss of generality, we may replace " $\stackrel{d}{=}$ by " $=$ " in the above equation. Thus we have

$$
\mathcal{L}_{t}\left(x^{n+2}\right)+\frac{n+2}{2} \int_{0}^{t} \mathcal{L}_{s}\left(x^{n+2}\right) d s=\mathcal{L}_{0}\left(x^{n+2}\right)+G_{t}\left(x^{n+2}\right)+R_{t}(n),
$$

whose solution is given by (3.39).

The proof is concluded.

Now we extend the result of Theorem 3.11 to a generalized system of (3.38).

\section{Corollary 3.12. Consider the following SDES}

$$
d \lambda_{i}^{N}(t)=\frac{1}{\sqrt{N}} d W_{i}(t)+\left(b_{N}\left(\lambda_{i}^{N}(t)\right)+\frac{1}{2 N} \sum_{j: j \neq i} \frac{1}{\lambda_{i}^{N}(t)-\lambda_{j}^{N}(t)}\right) d t, 1 \leq i \leq N, t \geq 0,
$$

where $b_{N}(x)$ satisfies, for some constant $c \in \mathbb{R}$,

$$
\lim _{N \rightarrow \infty} N\left\|b_{N}(x)+\frac{1}{2} x-c\right\|_{L^{\infty}(\mathbb{R})}=0 .
$$

Furthermore, assume the same initial conditions as in Theorem 3.11. Then the conclusion of Theorem 3.11 still holds with $R_{t}(n)$ in (3.40) replaced by

$$
\begin{aligned}
R_{t}(n) & =c(n+2) \int_{0}^{t} \mathcal{L}_{s}^{N}\left(x^{n+1}\right) d s+\frac{(n+2)(n+1)}{4} \int_{0}^{t}\left\langle x^{n}, \mu_{s}\right\rangle d s \\
& +\frac{n+2}{2} \sum_{k=0}^{n} \int_{0}^{t} \mathcal{L}_{s}\left(x^{n-k}\right)\left\langle x^{k}, \mu_{s}\right\rangle d s .
\end{aligned}
$$

Proof. The proof is similar to the proof of Corollary 3.10, which is sketched below.

By (3.47), without loss of generality, we assume

$$
-\frac{1}{2} x+c-1 \leq b_{N}(x) \leq-\frac{1}{2} x+c+1,
$$


for all $N \geq 1$. Then we have

$$
\mathbb{P}\left(x_{i}^{N}(t) \leq \lambda_{i}^{N}(t) \leq y_{i}^{N}(t), \forall 1 \leq i \leq N, \forall t>0\right)=1,
$$

where the processes $\left(x_{i}^{N}(t)\right)_{1 \leq i \leq N}$ and $\left(y_{i}^{N}(t)\right)_{1 \leq i \leq N}$ are the solutions of the following systems of SDEs respectively:

$d x_{i}^{N}(t)=\frac{1}{\sqrt{N}} d W_{i}(t)+\left(-\frac{1}{2} x_{i}^{N}(t)+c-1+\frac{1}{2 N} \sum_{j: j \neq i} \frac{1}{x_{i}^{N}(t)-x_{j}^{N}(t)}\right) d t, 1 \leq i \leq N, t \geq 0$,

and

$d y_{i}^{N}(t)=\frac{1}{\sqrt{N}} d W_{i}(t)+\left(-\frac{1}{2} y_{i}^{N}(t)+c+1+\frac{1}{2 N} \sum_{j: j \neq i} \frac{1}{y_{i}^{N}(t)-y_{j}^{N}(t)}\right) d t, 1 \leq i \leq N, t \geq 0$,

with the initial conditions $x_{i}^{N}(0)=y_{i}^{N}(0)=\lambda_{i}^{N}(0)$ for $1 \leq i \leq N$. Noting that $\left(x_{i}^{N}(t)-\right.$ $2 c+2)_{1 \leq i \leq N}$ and $\left(y_{i}^{N}(t)-2 c-2\right)_{1 \leq i \leq N}$ solve the SDEs (3.38), by (3.45) and (3.48), we get that the uniform $L^{p}$ bound (2.15) holds for system (3.46).

Then applying Corollary 2.5 and following the approach in the proof of Theorem 3.5, we get the desired result.

\section{A Some lemmas}

In this section, we provide some results that were used in the preceding sections.

The following CLT for martingales was used in the proof of Theorem 2.2.

Lemma A.1 (Rebolledo's Theorem). Let $n \in \mathbb{N}$, and let $\left\{M_{N}\right\}_{N \in \mathbb{N}}$ be a sequence of continuous centered martingales with values in $\mathbb{R}^{n}$. If the quadratic variation $\left\langle M_{N}\right\rangle_{t}$ converges in $L^{1}(\Omega)$ to a continuous deterministic function $\phi(t)$ for all $t>0$, then for any $T>0$, as a continuous process from $[0, T]$ to $\mathbb{R}^{n},\left(M_{N}(t), t \in[0, T]\right)$ converges in law to a Gaussian process $G$ with mean 0 and covariance

$$
\mathbb{E}\left[G(s) G(t)^{\top}\right]=\phi(t \wedge s) .
$$

Section 3.1 was based on the following comparison principle for multi-dimensional SDEs which is a direct consequence of (Geiß and Manthey, 1994, Theorem 1.1 and Theorem 1.2).

Lemma A.2. On a certain complete probability space equipped with a filtration that satisfies the usual conditions ((Karatzas and Shreve, 1991, Definition 2.25)), consider the following SDEs

$$
\begin{aligned}
& Y(t)=Y(0)+\int_{0}^{t} b^{(1)}(s, Y(s)) d s+\int_{0}^{t} \sigma(s, Y(s)) d W(s), \\
& Z(t)=Z(0)+\int_{0}^{t} b^{(2)}(s, Z(s)) d s+\int_{0}^{t} \sigma(s, Z(s)) d W(s),
\end{aligned}
$$

where $\{W(t), t \geq 0\}$ is a $d$-dimensional Brownian motion. Assume the solutions to SDEs (A.1) are pathwisely unique and non-exploding. If the following conditions are satisfied,

1. the drift functions $b^{(1)}(t, x)$ and $b^{(2)}(t, x)$ are continuous mappings from $[0, \infty) \times \mathbb{R}^{n}$ to $\mathbb{R}^{n}$. Besides, they are quasi-monotonously increasing in the sense that for $1 \leq i \leq n$ and $j=1,2, b_{i}^{(j)}(t, x) \leq b_{i}^{(j)}(t, y)$, whenever $x_{i}=y_{i}$ and $x_{l} \leq y_{l}$ for $l \in\{1, \ldots, n\} \backslash\{i\}$; 
2. the dispersion matrix $\sigma(t, x)$ is a continuous mapping from $[0, \infty) \times \mathbb{R}^{n}$ to $\mathbb{R}^{n \times d}$ that satisfies the following condition

$$
\sum_{j=1}^{d}\left|\sigma_{i j}(t, x)-\sigma_{i j}(t, y)\right| \leq \rho\left(\left|x_{i}-y_{i}\right|\right)
$$

for all $t \geq 0$ and $x=\left(x_{1}, \ldots, x_{n}\right)^{\top}, y=\left(y_{1}, \ldots, y_{n}\right)^{\top} \in \mathbb{R}^{n}$, where $\rho:[0, \infty) \rightarrow[0, \infty)$ is a strictly increasing function with $\rho(0)=0$ and

$$
\int_{0+} \rho^{-2}(u) d u=\infty
$$

3. $b_{i}^{(1)}(t, x) \leq b_{i}^{(2)}(t, x)$ for all $1 \leq i \leq n, t \geq 0, x \in \mathbb{R}^{n}$;

4. for $1 \leq i \leq n, Y_{i}(0) \leq Z_{i}(0)$ almost surely,

then we have

$$
\mathbb{P}\left(Y_{i}(t) \leq Z_{i}(t), \forall t \geq 0,1 \leq i \leq n\right)=1 .
$$

The following lemma was employed in the proof of Lemma 3.3.

Lemma A.3. Let $u^{N}(t)$ be the strong solution to (3.8). If $u^{N}(t)$ is distributed according to $\mathbb{P}^{N}$ in (3.7), then for $f \in C_{b}^{2}\left(\mathbb{R}^{N}\right)$,

$$
\frac{d}{d t} \mathbb{E}\left[f\left(u^{N}(t)\right)\right]=0
$$

Proof. For $f \in C_{b}^{2}\left(\mathbb{R}^{N}\right)$, applying Itô's formula to (3.8), we have

$$
\begin{aligned}
f\left(u^{N}(t)\right)= & f\left(u^{N}\left(t_{0}\right)\right)+\sum_{i=1}^{N} \int_{0}^{t} \partial_{i} f\left(u^{N}(s)\right) \cdot 2 \frac{\sqrt{u_{i}^{N}(s)}}{\sqrt{N(s+a)}} d W_{i}(s) \\
& +\sum_{i=1}^{N} \int_{0}^{t} \partial_{i} f\left(u^{N}(s)\right) \cdot \frac{1}{s+a}\left(\frac{P}{N}-u_{i}^{N}(s)+\frac{1}{N} \sum_{j: j \neq i} \frac{u_{i}^{N}(s)+u_{j}^{N}(s)}{u_{i}^{N}(s)-u_{j}^{N}(s)}\right) d s \\
& +\frac{1}{2} \sum_{i=1}^{N} \int_{0}^{t} \partial_{i}^{2} f\left(u^{N}(s)\right) \cdot 4 \frac{u_{i}^{N}(s)}{N(s+a)} d s .
\end{aligned}
$$

Here, $\partial_{i}$ is the partial derivative with respect to the $i$-th component $x_{i}$. Therefore, for $t \geq 0$,

$$
\begin{aligned}
\frac{d}{d t} \mathbb{E}\left[f\left(u^{N}(t)\right)\right]= & \mathbb{E}\left[\frac{1}{t+a} \sum_{i=1}^{N} \partial_{i} f\left(u^{N}(t)\right) \cdot\left(\frac{P}{N}-u_{i}^{N}(t)\right)\right] \\
& +\mathbb{E}\left[\frac{1}{N(t+a)} \sum_{i \neq j} \partial_{i} f\left(u^{N}(t)\right) \cdot \frac{u_{i}^{N}(t)+u_{j}^{N}(t)}{u_{i}^{N}(t)-u_{j}^{N}(t)}\right] \\
& +\mathbb{E}\left[\frac{2}{N(t+a)} \sum_{i=1}^{N} \partial_{i}^{2} f\left(u^{N}(t)\right) u_{i}^{N}(t)\right] .
\end{aligned}
$$

Thus it suffices to show, with the density function $p(x)$ in (3.7),

$$
\begin{aligned}
& \sum_{i=1}^{N} \int_{\Delta_{N}} \partial_{i} f(x) \cdot\left(\frac{P}{N}-x_{i}\right) p(x) d x+\frac{1}{N} \sum_{i \neq j} \int_{\Delta_{N}} \partial_{i} f(x) \cdot \frac{x_{i}+x_{j}}{x_{i}-x_{j}} p(x) d x \\
& +\frac{2}{N} \sum_{i=1}^{N} \int_{\Delta_{N}} \partial_{i}^{2} f(x) x_{i} p(x) d x=0,
\end{aligned}
$$


where $\Delta_{N}=\left\{x \in \mathbb{R}^{N}: 0<x_{1}<\ldots<x_{N}\right\}$ is the support of $\mathbb{P}^{N}$. Noting that $p(x)$ vanishes on $\partial \Delta_{N}$, we have by the integration by parts formula,

$$
\begin{aligned}
\int_{\Delta_{N}} \partial_{i}^{2} f(x) x_{i} p(x) d x & =\int_{\partial \Delta_{N}} \partial_{i} f(x) x_{i} p(x) d S-\int_{\Delta_{N}} \partial_{i} f(x) \partial_{i}\left(x_{i} p(x)\right) d x \\
& =-\int_{\Delta_{N}} \partial_{i} f(x)\left(p(x)+x_{i} \partial_{i} p(x)\right) d x .
\end{aligned}
$$

Hence, to show (A.2), it is sufficient to verify

$$
\sum_{i=1}^{N}\left(\frac{P}{N}-x_{i}\right) p(x)+\frac{1}{N} \sum_{i \neq j} \frac{x_{i}+x_{j}}{x_{i}-x_{j}} p(x)-\frac{2}{N} \sum_{i=1}^{N}\left(p(x)+x_{i} \partial_{i} p(x)\right)=0 .
$$

By the chain rule,

$$
\partial_{i} p(x)=-\frac{N}{2} p(x)+\frac{P-N-1}{2} \frac{1}{x_{i}} p(x)+\sum_{j: j \neq i} \frac{1}{x_{i}-x_{j}} p(x) .
$$

Hence,

$$
\begin{aligned}
\frac{2}{N} \sum_{i=1}^{N} x_{i} \partial_{i} p(x) & =-\sum_{i=1}^{N} x_{i} p(x)+(P-N-1) p(x)+\frac{2}{N} \sum_{i \neq j} \frac{x_{i}}{x_{i}-x_{j}} p(x) \\
& =-\sum_{i=1}^{N} x_{i} p(x)+(P-N-1) p(x)+\frac{1}{N} \sum_{i \neq j}\left(\frac{x_{i}+x_{j}}{x_{i}-x_{j}}+1\right) p(x) \\
& =-\sum_{i=1}^{N} x_{i} p(x)+(P-2) p(x)+\frac{1}{N} \sum_{i \neq j} \frac{x_{i}+x_{j}}{x_{i}-x_{j}} p(x),
\end{aligned}
$$

which gives the desired result.

Acknowledgments. The authors are grateful to the referees for their valuable and detailed comments.

\section{References}

Anderson, G. W., Guionnet, A., and Zeitouni, O. (2010). An Introduction to Random Matrices. Cambridge University Press, Cambridge New York. MR-2760897

Bru, M.-F. (1991). Wishart processes. J. Theor. Probab, 4(4):725-751. MR-1132135

Cabanal-Duvillard, T. (2001). Fluctuations de la loi empirique de grandes matrices aléatoires. Ann. Inst. H. Poincaré Probab. Statist., 37(3):373-402. MR-1831988

Cabanal-Duvillard, T. and Guionnet, A. (2001). Large deviations upper bounds for the laws of matrix-valued processes and non-communicative entropies. Ann. Probab., 29(3):1205-1261. MR-1872742

Cépa, E. and Lépingle, D. (1997). Diffusing particles with electrostatic repulsion. Probab. Theory Related Fields, 107(4):429-449. MR-1440140

Chan, T. (1992). The Wigner semi-circle law and eigenvalues of matrix-valued diffusions. Probab. Theory Related Fields, 93(2):249-272. MR-1176727

Dyson, F. J. (1962). A Brownian-motion model for the eigenvalues of a random matrix. J. Mathematical Phys., 3:1191-1198. MR-0148397

Fontbona, J. (2004). Uniqueness for a weak nonlinear evolution equation and large deviations for diffusing particles with electrostatic repulsion. Stochastic Process. Appl., 112(1):119-144. MR-2062570 
High-dimensional CLTs for a class of particle systems

Geiß, C. and Manthey, R. (1994). Comparison theorems for stochastic differential equations in finite and infinite dimensions. Stochastic Process. Appl., 53(1):23-35. MR-1290705

Graczyk, P. and Małecki, J. (2013). Multidimensional Yamada-Watanabe theorem and its applications to particle systems. J. Math. Phys., 54(2):021503, 15. MR-3076363

Graczyk, P. and Małecki, J. (2014). Strong solutions of non-colliding particle systems. Electron. J. Probab., 19:no. 119, 21. MR-3296535

Graczyk, P. and Małecki, J. (2019). On squared Bessel particle systems. Bernoulli, 25(2):828-847. MR-3920358

Hiai, F. and Petz, D. (2000). The semicircle law, free random variables and entropy, volume 77 of Mathematical Surveys and Monographs. American Mathematical Society, Providence, RI. MR-1746976

Karatzas, I. and Shreve, S. E. (1991). Brownian Motion and Stochastic Calculus, volume 113 of Graduate Texts in Mathematics. Springer-Verlag, New York, second edition. MR-1121940

Li, S., Li, X.-D., and Xie, Y.-X. (2020). On the law of large numbers for the empirical measure process of generalized Dyson Brownian motion. J. Stat. Phys., 181(4):1277-1305. MR-4163502

Małecki, J. and Pérez, J. L. (2019). Universality classes for general random matrix flows. arXiv e-prints, page arXiv:1901.02841.

Mingo, J. A. and Speicher, R. (2017). Free probability and random matrices, volume 35 of Fields Institute Monographs. Springer, New York; Fields Institute for Research in Mathematical Sciences, Toronto, ON. MR-3585560

Pérez-Abreu, V. and Tudor, C. (2007). Functional limit theorems for trace processes in a Dyson Brownian motion. Commun. Stoch. Anal., 1(3):415-428. MR-2403859

Perez-Abreu, V. and Tudor, C. (2009). On the traces of Laguerre processes. Electron. J. Probab., 14:no. 76, 2241-2263. MR-2550298

Rogers, L. C. G. and Shi, Z. (1993). Interacting Brownian particles and the Wigner law. Probab. Theory Related Fields, 95(4):555-570. MR-1217451

Rudin, W. (1991). Functional Analysis. International Series in Pure and Applied Mathematics. McGraw-Hill, Inc., New York, second edition. MR-1157815

Song, J., Yao, J., and Yuan, W. (2020). High-dimensional limits of eigenvalue distributions for general Wishart process. Ann. Appl. Probab., 30(4):1642-1668. MR-4132637

Voiculescu, D. (1991). Limit laws for random matrices and free products. Invent. Math., 104(1):201220. MR-1094052

Voiculescu, D. V., Dykema, K. J., and Nica, A. (1992). Free random variables, volume 1 of CRM Monograph Series. American Mathematical Society, Providence, RI. A noncommutative probability approach to free products with applications to random matrices, operator algebras and harmonic analysis on free groups. MR-1217253 


\section{Electronic Journal of Probability Electronic Communications in Probability}

\section{Advantages of publishing in EJP-ECP}

- Very high standards

- Free for authors, free for readers

- Quick publication (no backlog)

- Secure publication $\left(\mathrm{LOCKSS}^{1}\right)$

- Easy interface (EJMS²)

\section{Economical model of EJP-ECP}

- Non profit, sponsored by $\mathrm{IMS}^{3}, \mathrm{BS}^{4}$, ProjectEuclid ${ }^{5}$

- Purely electronic

\section{Help keep the journal free and vigorous}

- Donate to the IMS open access fund ${ }^{6}$ (click here to donate!)

- Submit your best articles to EJP-ECP

- Choose EJP-ECP over for-profit journals

\footnotetext{
${ }^{1}$ LOCKSS: Lots of Copies Keep Stuff Safe http://www. lockss.org/

${ }^{2}$ EJMS: Electronic Journal Management System http://www.vtex.lt/en/ejms.html

${ }^{3}$ IMS: Institute of Mathematical Statistics http://www.imstat.org/

${ }^{4}$ BS: Bernoulli Society http://www. bernoulli-society.org/

${ }^{5}$ Project Euclid: https://projecteuclid.org/

${ }^{6}$ IMS Open Access Fund: http://www.imstat.org/publications/open.htm
} 